\title{
LYAPUNOV-LIKE CONDITIONS OF FORWARD INVARIANCE AND BOUNDEDNESS FOR A CLASS OF UNSTABLE SYSTEMS*
}

\author{
A. GORBAN $^{\dagger}$, I. TYUKIN ${ }^{\ddagger}$, E. STEUR ${ }^{\S}$, AND H. NIJMEIJER
}

\begin{abstract}
We provide Lyapunov-like characterizations of boundedness and convergence of nontrivial solutions for a class of systems with unstable invariant sets. Examples of systems to which the results may apply include interconnections of stable subsystems with one-dimensional unstable dynamics or critically stable dynamics. Systems of this type arise in problems of nonlinear output regulation, parameter estimation, and adaptive control. In addition to providing boundedness and convergence criteria, the results allow us to derive domains of initial conditions corresponding to solutions leaving a given neighborhood of the origin at least once. In contrast to other works addressing convergence issues in unstable systems, our results require neither input-output characterizations for the stable part nor estimates of convergence rates. The results are illustrated with examples, including the analysis of phase synchronization of neural oscillators with heterogeneous coupling.
\end{abstract}

Key words. convergence, weakly attracting sets, Lyapunov functions, synchronization

AMS subject classifications. 34C11, 34D05, 34E18, 37B25, 37C70, 93C10, 93C41

DOI. $10.1137 / 120865173$

1. Introduction. Methods and tools for the analysis of asymptotic properties of solutions of ordinary differential equations are important components of modern control theory. Even though the problems of control are often viewed as that of synthesis rather than analysis, the latter crucially affects the former. Indeed, in order to be able to specify feasible goals of synthesis, e.g., forward-completeness, state boundedness, asymptotic convergence of solutions to a region in the state space, etc., one needs to understand how these properties depend on the system parameters and controls.

The majority of the analysis techniques in control, and hence methods for systems design, rely upon the assumption that desired motions in the system are stable in the sense of Lyapunov [23]. Let us briefly recall this and other related notions from the domain of dynamical systems and also introduce notational conventions used throughout the manuscript.

1.1. Notation and basic notions. The following notational conventions are used throughout the paper. Let $\mathcal{D}$ be an open set in $\mathbb{R}^{n}$. The symbol $\mathcal{C}^{k}(\mathcal{D})$ denotes the space of functions that are at least $k$ times differentiable in $\mathcal{D} ; \overline{\mathcal{D}}$ denotes the closure of $\mathcal{D} ;\|\cdot\|$ stands for the Euclidean norm. Let $\mathcal{S}$ be a subset of $\mathbb{R}^{n}$, and let $x \in \mathbb{R}^{n}$; then $\operatorname{dist}(\mathcal{S}, x)=\inf _{x^{\prime} \in \mathcal{S}}\left\|x-x^{\prime}\right\|$. By $\mathcal{K}_{0}$ we denote the set of all nondecreasing continuous functions $\kappa: \mathbb{R}_{\geq 0} \rightarrow \mathbb{R}_{\geq 0}$ such that $\kappa(0)=0 ; \mathcal{K} \subset \mathcal{K}_{0}$ is the subset of strictly increasing functions, and $\mathcal{K}_{\infty} \subset \mathcal{K}$ consists of functions from $\mathcal{K}$ with infinite limit: $\lim _{s \rightarrow \infty} \kappa(s)=\infty$. Consider a nonautonomous system $\dot{x}=f(x, p, t)$,

${ }^{*}$ Received by the editors February 8, 2012; accepted for publication (in revised form) February 28, 2013; published electronically

$\dagger$ Department of Mathematics, University of Leicester, Leicester, LE1 7RH, UK (ag153@le.ac.uk).

${ }^{\ddagger}$ Corresponding author. Department of Mathematics, University of Leicester, Leicester, LE1 7RH, UK (I.Tyukin@le.ac.uk).

$\S$ Laboratory for Perceptual Dynamics, KU Leuven, 3000 Leuven, Belgium (erik.steur@ppw. kuleuven.be).

"Department of Mechanical Engineering, Eindhoven University of Technology, Eindhoven, The Netherlands (h.nijmeijer@tue.nl). 
where $f: \mathbb{R}^{n} \times \mathbb{R}^{d} \times \mathbb{R} \rightarrow \mathbb{R}^{n}$ is continuous, $p \in \mathbb{R}^{d}$ is the vector of parameters, and $f(\cdot, p, t)$ is locally Lipschitz; $x\left(\cdot ; t_{0}, x_{0} \mid p\right)$ stands for the unique maximal solution of the initial value problem: $x\left(t_{0} ; t_{0}, x_{0} \mid p\right)=x_{0}$. In cases when no confusion arises, we will refer to these solutions as $x\left(\cdot ; t_{0}, x_{0}\right), x\left(\cdot ; x_{0}\right)$, or simply $x(\cdot)$. Solutions of the initial value problem above at $t$ are denoted as $x\left(t ; t_{0}, x_{0}\right), x\left(t ; x_{0}\right)$, or $x(t)$, respectively. We always separate by the semicolon the symbol of the independent (time) variable from symbols of other variables (initial data or parameters).

Let us start with the classical notion of invariance of a set. Let $\mathcal{D}$ be an open subset of $\mathbb{R}^{n}$, and consider systems represented by differential equations $\dot{x}=f(x)$ in the domain $\mathcal{D}$. The right-hand side, $f(x)$, is assumed to be a locally Lipschitz vector-field on $\mathcal{D}$. In this case, for any initial condition $x(0)=x_{0}, x_{0} \in \mathcal{D}$, the system has a solution $x\left(\cdot ; x_{0}\right)$ defined on a time interval $(-\tau, \tau)$ where $\tau>0$ may depend on $x_{0}$. A set $\mathcal{S}, \mathcal{S} \subset \mathcal{D}$, is forward invariant (w.r.t. the system dynamics) if for every $x_{0} \in \mathcal{S}, x\left(\cdot ; x_{0}\right)$ is defined on $[0, \infty)$ and $x\left(t ; x_{0}\right) \in \mathcal{S}$ for all $t>0$. $\mathcal{S}$ is invariant if for every $x_{0} \in \mathcal{S}$ the solution $x\left(\cdot ; x_{0}\right)$ is defined on $(-\infty, \infty)$, and $x\left(t ; x_{0}\right) \in \mathcal{S}$ for all $t \in \mathbb{R}$. Unions and intersections of a family of (forward) invariant sets are (forward) invariant.

A closed invariant set $\mathcal{S} \subset \mathcal{D}$ is a weakly attracting set if there exists a set $\mathcal{V} \subset \mathcal{D}$ with strictly positive measure such that for all $x_{0} \in \mathcal{V}$ the solution $x\left(\cdot ; x_{0}\right)$ is defined on $[0, \infty)$ and the following holds: $\lim _{t \rightarrow \infty} \operatorname{dist}\left(\mathcal{S}, x\left(t ; x_{0}\right)\right)=0[26]$. The set $\mathcal{V}$ is not necessarily a neighborhood of $\mathcal{S}$. The set is attracting if $\mathcal{V}$ is a neighborhood of $\mathcal{S}$, and $\mathcal{V}$ is forward invariant. A closed invariant set $\mathcal{S} \subset \mathcal{D}$ is stable in the sense of Lyapunov if for any neighborhood $\mathcal{V}$ of $\mathcal{S}$ there exists a forward invariant neighborhood $\mathcal{W} \subset \mathcal{D}$ of $\mathcal{S}$ such that $\mathcal{W} \subset \mathcal{V}[41]$. In other words, a set that is stable in the sense of Lyapunov has a fundamental base of forward invariant neighborhoods. (A collection $\mathcal{U}_{\mathcal{S}}$ of all neighborhoods of $\mathcal{S}$ is called a neighborhood system of $\mathcal{S}$. A subcollection $\mathcal{B}_{\mathcal{S}} \subset \mathcal{U}_{\mathcal{S}}$ is a fundamental base of system $\mathcal{U}_{\mathcal{S}}$ iff every element of $\mathcal{U}_{\mathcal{S}}$ contains at least one element of $\mathcal{B}_{\mathcal{S}}$.)

For noncompact sets $\mathcal{S} \subset \mathcal{D}$ it may be useful to distinguish the notion of Lyapunov stability from the notion of uniform Lyapunov stability that is defined with uniform neighbourhoods [10].

Various extensions of stability of sets are proposed for nonautonomous systems too [20]. For these systems we need the notion of forward invariance of sets in the state space. Consider systems $\dot{x}=f(x, t)$ in a domain $\mathcal{D} \times \mathbb{R} \subset \mathbb{R}^{n+1}$, where the vector-field $f: \mathcal{D} \times \mathbb{R} \rightarrow \mathbb{R}^{n}$ is continuous, and $f(\cdot, t)$ is locally Lipschitz uniformly in $t$. A set $\mathcal{S} \subset \mathcal{D}$ is $t_{0}$-forward invariant w.r.t. dynamics if for given $t_{0} \in \mathbb{R}$ and every $x_{0} \in \mathcal{S}$ the solution $x\left(\cdot ; t_{0}, x_{0}\right)$ is defined on $\left[t_{0}, \infty\right)$ and $x\left(t ; t_{0}, x_{0}\right) \in \mathcal{S}$ for $t \geq t_{0}$. If it is $t_{0}$-forward invariant for all $t_{0}$, then we call it forward invariant. In this work, we use systems of nested forward invariant sets to characterize the attractivity of solutions that are not stable in the classical senses.

The notion of Lyapunov stability and analysis methods that are based on this notion are proven successful in a wide range of engineering applications (see as a list of nonexhaustive references, e.g., [30], [18], [22], [29]). The popularity and success of the concept of Lyapunov stability resides, to a substantial degree, in the convenience and utility of the method of Lyapunov functions for assessing asymptotic properties of solutions of ordinary differential equations. Instead of deriving the solutions explicitly it suffices to solve an algebraic inequality involving partial derivatives of a given Lyapunov candidate function.

As the methods of control expand from purely engineering applications into a wider area of science, there is a need for maintaining behavior that fails to obey the 

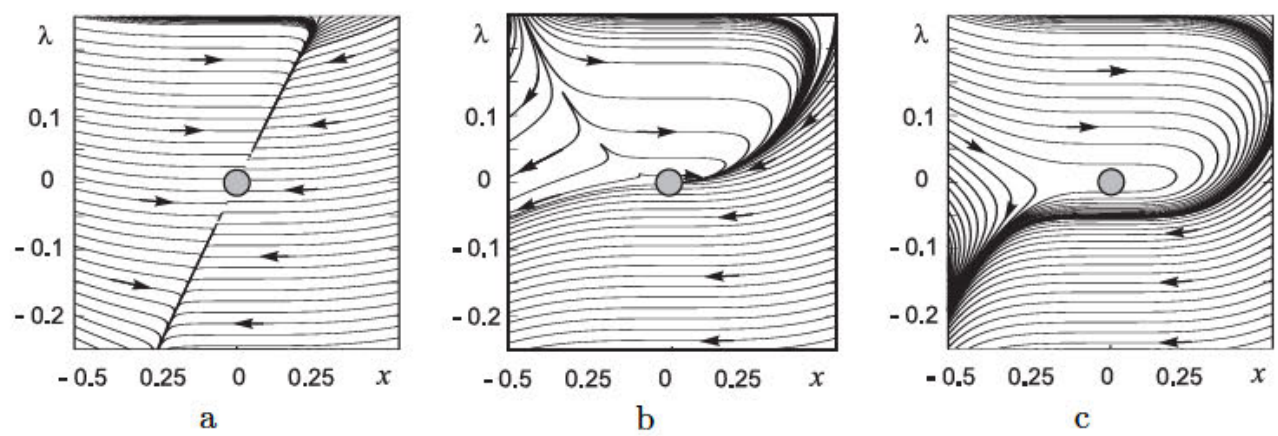

FIG. 1. Phase plots of (1a) (panel a), (1b) (panel b), and (1c) (panel c) for $\gamma=0.5$.

usual requirement of Lyapunov stability. There are numerous examples of systems possessing Lyapunov-unstable, yet attracting, invariant sets [2], e.g., in the domains of aircraft dynamics and the design of synchronous generators [6, pp. 313-356]. Even though solutions of these systems may not always be uniformly asymptotically stable, they are required to be bounded and converging to some specified areas in the system state space. Finding rigorous and tight criteria for asymptotic convergence to Lyapunov-unstable invariant sets, however, is a nontrivial problem.

1.2. Motivating examples. Let us start from examples showing nontrivialities of asymptotic behavior of solutions in systems with unstable invariant sets.

Example 1. Consider the following systems:

$$
\left\{\begin{array}{l}
\dot{x}=-x+\lambda, \\
\dot{\lambda}=-\gamma|x|^{3},
\end{array}\right.
$$

$$
\left\{\begin{array}{l}
\dot{x}=-x^{2}+\lambda \\
\dot{\lambda}=-\gamma|x|^{3}
\end{array}\right.
$$

$$
\left\{\begin{array}{l}
\dot{x}=-x^{3}+\lambda \\
\dot{\lambda}=-\gamma|x|^{3}
\end{array}\right.
$$

Phase plots of these systems are shown in Figure 1. Systems (1a), (1c) share an apparent similarity in their description. Indeed, $x=0$ is the unique asymptotically stable equilibrium of their first equations at $\lambda=0$, and their second equations governing the dynamics of variable $\lambda$ are identical. System (1b), to the contrary, is fundamentally different from (1a), (1c): the equilibrium $x=0$ of its first equation is not stable at $\lambda=0$. Thus one could expect qualitative similarity of the dynamics of (1a) and (1c), whereas no such similarity is expected between (1b) and (1a). However, as the phase plots suggest (see Figure 1), the origins of (1a), (1b) are weak attractors, and the origin of (1c) is not an attractor. This can be demonstrated, for example, by using the following singular transformation: $(x, \lambda) \mapsto(\rho, \varphi), x=\rho \cos \varphi, \lambda=\rho^{2} \sin (\varphi)$ (for details see [8]). Therefore, our initial naive intuition about qualitative properties of solutions of (1a)-(1c) failed. This motivates the necessity for having rigorous, simple, and efficient criteria for assessing asymptotic properties of solutions in systems with unstable invariant sets.

In pursuing this goal we do not wish to attempt addressing the issue in full generality, namely for the widest class of systems possible. Instead we focus on a particular family of equations that occur naturally in a range of control, optimization, estimation, and modeling problems. Examples of systems from this family are provided below.

Example 2. A network of phase oscillators provides an example of systems in which unstable attractors are shown (numerically) to exist and sometimes prevail [35], [36]. One of the simplest instances of such networks is a network of three phase 
oscillators of which the phase differences are governed by the following set of equations (see section 4.2):

$$
\begin{aligned}
& \dot{\vartheta}_{1}=\varepsilon / \pi \sin \left(\vartheta_{2}\right)^{2} \\
& \dot{\vartheta}_{2}=-\varepsilon_{1} / 2 \sin \left(2 \vartheta_{2}\right)+\varepsilon / \pi\left(\sin \left(\vartheta_{1}\right)^{2}+\sin \left(\vartheta_{2}\right)^{2}\right) .
\end{aligned}
$$

Variables $\vartheta_{1}, \vartheta_{2}$ denote the phase differences, and $\varepsilon, \varepsilon_{1}$ are parameters representing the coupling strengths between oscillators. It is clear that the origin of $(2 \mathrm{a}),(2 \mathrm{~b})$ is unstable in the sense of Lyapunov. This rules out explicit application of Lyapunov function-based approaches for deriving conditions of synchronization in such systems. The questions, nevertheless, are, For which values of $\varepsilon, \varepsilon_{1}$ will phase synchronization occur, how large is the domain of initial conditions leading to such a synchronous state, and how does it depend on $\varepsilon, \varepsilon_{1}$ ?

Example 3. A universal adaptive stabilizer of nonlinear systems (in the presence of uncertainties). The studied question is as follows. Consider the following system:

$$
\begin{aligned}
& \dot{x}=f(x, t)+g(x, t) u(t), f: \mathbb{R}^{n} \times \mathbb{R} \rightarrow \mathbb{R}^{n}, g: \mathbb{R}^{n} \times \mathbb{R} \rightarrow \mathbb{R}^{n \times m}, \\
& y=h(x), h: \mathbb{R}^{n} \rightarrow \mathbb{R}^{d},
\end{aligned}
$$

where $y$ is the output, and $u: \mathbb{R} \rightarrow \mathbb{R}^{m}$ is a control input which may depend on the current value of $y(t)=h(x(t))$ and on time $t$ directly. The functions $f(\cdot, \cdot), g(\cdot, \cdot)$ are not known explicitly. What a priori information about the system should be made available in order to derive a control input $u(\cdot)$ stabilizing the zero solutions of (3)? The question has been answered in [15], [31] (see also [1], [17], [16] for related work). Assume that there exists a matrix $K \in \mathbb{R}^{m \times d}$ such that the zero solution of $\dot{x}=f(x, t)+g(x, t) K h(x)$ is exponentially stable. The following system with the stabilizer was constructed:

$$
\begin{aligned}
& \dot{x}=f(x, t)+g(x, t) \beta(\gamma(\lambda)) h(x), \\
& \dot{\lambda}=\|h(x)\|^{p}, p \geq 1,
\end{aligned}
$$

where $\beta(\cdot): \mathbb{R} \rightarrow \mathbb{R}^{m \times d}$ is a special function with dense image in $\mathbb{R}^{m \times d}$ and $\gamma: \mathbb{R} \rightarrow \mathbb{R}$ is a special monotone function whose growth rate decays to zero as $\lambda \rightarrow \infty$ [15]:

$$
\lim _{\lambda \rightarrow \infty} \lambda \cdot \sup _{\ell \geq \lambda}\left|\frac{d}{d \ell} \gamma(\ell)\right| \cdot \sup _{\ell \in[0, \lambda]}\|\beta(\gamma(\ell))\|^{p}=0 .
$$

It was proven that the $x$-component of solutions converges to the origin as $t \rightarrow \infty$. (For the details of $\beta(\cdot)$ and $\gamma(\cdot)$ construction and specific conditions on functions $f(\cdot, \cdot)$, $g(\cdot, \cdot)$, and $h(\cdot)$, see the original papers.) Nevertheless, solutions of the extended system (4) are not uniformly asymptotically stable (cf. [37]).

Despite the problems described in Examples 2 and 3 arising in different subject areas, they are inherently similar. In both cases we have to deal with systems composed of an "attracting" subsystem coupled with a "wandering" one. The attracting subsystem has an attracting invariant set in its state space, and solutions of the wandering subsystem unidirectionally evolve along a certain path. The general description of the composed system is provided below. 
1.3. Class of systems. We will focus on systems whose dynamics can be described by the system of ordinary differential equations

$$
\left\{\begin{array}{l}
\dot{x}=f(x, \lambda, t), \\
\dot{\lambda}=g(x, \lambda, t),
\end{array}\right.
$$

where the vector-fields $f: \mathbb{R}^{n} \times \mathbb{R} \times \mathbb{R} \rightarrow \mathbb{R}^{n}, g: \mathbb{R}^{n} \times \mathbb{R} \times \mathbb{R} \rightarrow \mathbb{R}$ are continuous, $f(\cdot, \cdot, t), g(\cdot, \cdot, t)$ are locally Lipschitz uniformly in $t$, and $g(\cdot, \cdot, \cdot)$ in (5a) is of constant sign. Equation (5a) describes the coupled system generalizing (1a)-(1c), (2a), (2b), and (4).

Throughout the paper we assume that $(0,0)$ is an equilibrium of $(5 \mathrm{a})$. Moreover, we assume that the origin $x=0$ is a weak attractor of the $x$-subsystem of (5a) for frozen values of $\lambda$ at $\lambda=0$; i.e., the origin $x=0$ of the system

$$
\left\{\begin{array}{l}
\dot{x}=f(x, \lambda, t) \\
\lambda=\text { const }, \lambda \in \mathbb{R}
\end{array}\right.
$$

is a weak attractor at $\lambda=0$. We also assume that for system (5b) there are a $p>0$ and a set $\underline{\omega}(p)$ which is forward invariant for all $\lambda \in[0, p]$ : if $x_{0} \in \underline{\omega}(p)$ and $\lambda \in[0, p]$, then

$$
x\left(t ; t_{0}, x_{0} \mid \lambda\right) \in \underline{\omega}(p) \quad \text { for all } t_{0} \in \mathbb{R}, t \geq t_{0} .
$$

In principle, $\underline{\omega}(p)$ is allowed to coincide with $\mathbb{R}^{n}$. Notice also that since we do not wish to impose any additional specific constraints (such as, e.g., minimality), the set $\underline{\omega}(p)$ is not uniquely determined by the system itself. For example, for the system $(5 \mathrm{~b})$ induced by the first equation of (1a) the sets $\underline{\omega}(p)$ can be chosen as intervals $\left[b_{1}, b_{2}\right], b_{1} \leq 0, b_{2} \geq p$, as well as $\left(-\infty, b_{2}\right],\left[b_{1}, \infty\right)$, or $(-\infty, \infty)$. For the system $(5 \mathrm{~b})$ corresponding to $(1 \mathrm{~b})$ the sets $\underline{\omega}(p)$ are $\left[0, b_{1}\right], b_{1} \geq \sqrt{p}$. For the sake of simplicity one may ignore references to $\underline{\omega}(p)$ in the statements of the results and assume that $\underline{\omega}(p)$ coincides with $\mathbb{R}^{n}$. On the other hand, as we shall see later, the introduction of $\underline{\omega}(p)$ enables us to produce criteria for checking whether an equilibrium is a weak attractor or not for dynamical systems (e.g., described by (1b)) in which the zero solution of (5b) at $\lambda=0$ is not stable in the sense of Lyapunov.

Further and specific technical assumptions about $f(\cdot, \cdot, \cdot)$ and $g(\cdot, \cdot, \cdot)$ are provided in section 2 .

Remark 1. If $\underline{\omega}(p)$ is forward invariant for (5b) for all $\lambda \in[0, p]$, then the set $\underline{\omega}(p)$ is also forward invariant w.r.t. equations

$$
\dot{x}=f(x, \lambda(t), t)
$$

for any piecewise-continuous function $\lambda(\cdot)$ with values $\lambda(t) \in[0, p]$ (and a discrete set of discontinuity points). This can be easily proved using approximation of $\lambda(\cdot)$ by piecewise constant functions.

In addition to the previous examples, equations (5a) describe estimation algorithms in problems of adaptive control and observer design when models of uncertainty are nonlinearly parameterized, or when the application of standard techniques is computationally ill-posed [11], [38]. They also can be viewed as a prototype for control and estimation schemes with prerouting in the domain of supervisory control [27].

A rather general interpretation of systems (5a) is that they govern a class of systems in which inherent dynamics of an object (first equation and (5b)) is coupled with the dynamics of the system's resources (second equation). In this regard $g(\cdot, \cdot, \cdot)$ defines the rate of the resource's consumption, and $f(\cdot, \cdot, \cdot)$ determines the velocity of the state $x$ given the available resources $\lambda$ at $t$. 
1.4. Extension of Lyapunov's idea onto unstable sets. The systems with inherently unstable behavior are important for many applications, including modeling, control, and identification (see, for example, [33], [32], where dissipative saddles were used to model decision-making sequences, [12], where the flutter suppressors were developed, and [15], [31], where the general problem of universal adaptive stabilization was studied). Nevertheless, there are limitations restricting further progress in application of the broader concept of unstable convergence in these areas. Among these is the lack of a simple analogue of the Lyapunov method for these, strictly speaking, unstable systems that would allow one to draw conclusions about asymptotic properties of unstable solutions without the need to solve the equations. This motivates the focus of our present work.

In this paper we propose an extension of the classical Lyapunov function method for assessing boundedness and convergence of motion in dynamical systems with unstable invariant sets. The class of systems we will consider is given by (5a), and the questions we address below can be formulated as follows:

1. Let the origin of (5a) be an equilibrium. Can we tell (without solving the system) whether the set is an attractor in some appropriate sense, e.g., Milnor's sense $[26]$ ?

2. Pick a point in the system's state space. Is it possible to predict (without solving the system) whether the solution passing through this point is bounded in forward time, or does it escape to infinity?

These questions are certainly not original. Algebraic criteria for checking attractivity of unstable point attractors in a rather general setting have been proposed in [34] and were further developed in [24], [40]. These results apply to systems in which almost all points in a neighborhood of the attractor correspond to solutions converging to the attractor asymptotically. Yet, as can be seen clearly from Figure 1, this requirement may not hold for the class of systems described by $(5 \mathrm{a})$. On the other hand, techniques which can be used to address the questions above for equations $(5 \mathrm{a})$, such as, e.g., [39], lack the convenience of the method of Lyapunov functions. Further, they require the existence of input-output gains for the stable subsystem. Hence, developing novel methods to address the issue of convergence to unstable sets is needed. These methods, on the one hand, should inherit the efficiency of Lyapunov analysis in which boundedness of solutions can be verified by checking a system of inequalities without involving prior knowledge of the solutions of the system. On the other hand, these methods should apply to systems with instabilities, such as those specified by (5a). In our present contribution we provide a set of results that can be considered as a possible candidate.

The main idea behind the development of these results can briefly be summarized as follows. Since we are interested in the solutions that are not necessarily stable in the sense of Lyapunov, we abandon the concept of neighborhoods from standard Lyapunov analysis [23], [41]. For a given invariant set $\mathcal{S}$ of a system, instead of searching for a fundamental base of forward invariant neighborhoods of $\mathcal{S}$, we study the existence of a collection of forward invariant sets associated with $\mathcal{S}$. These sets are not necessarily neighborhoods, and they are not required to form a fundamental base. In particular, the sets are allowed to be closed, and their boundaries may have nonempty intersections with $\mathcal{S}$.

For the chosen class of dynamical systems we formulate Lyapunov-like conditions that allow us to specify forward invariant sets containing Lyapunov-unstable equilibria on their boundaries. In the classical method of Lyapunov functions the role of a Lyapunov function is to ensure that an invariant set, e.g., an equilibrium, has a 
fundamental base of forward invariant neighborhoods. In our work we use an extension of this method in which a substitute of a Lyapunov function is used to demonstrate the existence of a family of forward invariant sets (not necessarily neighborhoods) associated with the equilibrium.

The method we use for determining positive invariance of an individual set is similar in spirit to the second method of Lyapunov [23] and its extensions [9], [41], [25], including equations with differential inclusions [5], [21], [28], [3], [4]. Namely, we are looking for closed sets containing the origin such that on the boundaries of these sets the vector-fields in the right-hand side of (5a) are pointing inward or vanishing. Following this intuition we demonstrate that there is a set of simple algebraic conditions, very similar to those in the second method of Lyapunov, enabling us to characterize asymptotic behavior of solutions for systems with unstable invariant sets. In particular, these results allow us to estimate the domains of initial conditions, as functions of system parameters, which are associated with bounded solutions in forward time without the need to require information about the convergence rate of the stable part of (5a). Parameters of these systems are not required to be known precisely, and input-output gains of the systems need not be defined. Furthermore, in contrast to our previous results on the same topic [39], the present conditions allow us to specify domains of initial conditions that lead to solutions necessarily escaping from a neighborhood of the equilibria in question.

The paper is organized as follows. In section 2 we formulate the problem and specify the main assumptions. Section 3 contains the main results of the paper.

Section 4 presents illustrative examples showing how the results can be applied to (1) derive estimates of attractor basins for (1a)-(1c), (2) solve the phase synchronization problem described in Example 2, and (3) design an adaptive control scheme for a class of systems with general nonlinear parametrization. Section 5 concludes the paper. Auxiliary technical results are presented in the appendix (section 6).

2. Problem formulation. Consider system (5a),

$$
\begin{aligned}
& \dot{x}=f(x, \lambda, t), \\
& \dot{\lambda}=g(x, \lambda, t),
\end{aligned}
$$

where the vector-fields $f: \mathbb{R}^{n} \times \mathbb{R} \times \mathbb{R} \rightarrow \mathbb{R}^{n}, g: \mathbb{R}^{n} \times \mathbb{R} \times \mathbb{R} \rightarrow \mathbb{R}$ are continuous and locally Lipschitz w.r.t. $x, \lambda$ uniformly in $t$. Recall that the point $x=0, \lambda=0$ is an equilibrium of (5a), that $x=0$ is a weak attractor for (5b) at $\lambda=0$, and that $\underline{\omega}(p), p>0$, is the set which is forward invariant for all $\lambda \in[0, p]$ w.r.t. the dynamics of $(5 b)$.

Let $\mathcal{D}$ be an open subset of $\mathbb{R}^{n}$, and let $\Lambda=\left[c_{1}, c_{2}\right], c_{1} \leq 0, c_{2}>0$, be an interval. Suppose that the closure $\overline{\mathcal{D}}$ of $\mathcal{D}$ contains the origin, and denote $\mathcal{D}_{\Omega}=\overline{\mathcal{D}} \times \Lambda \times \mathbb{R}$. Finally, we suppose that the right-hand side of (5a) satisfies Assumptions 1 and 2 below.

Assumption 1. There exist a function $V: \mathbb{R}^{n} \rightarrow \mathbb{R}, V \in \mathcal{C}^{0}$, differentiable everywhere except possibly at the origin, and five functions of one variable, $\underline{\alpha}, \bar{\alpha} \in \mathcal{K}_{\infty}$, $\alpha: \mathbb{R}_{\geq 0} \rightarrow \mathbb{R}, \alpha \in \mathcal{C}^{0}([0, \infty)), \alpha(0)=0, \beta: \mathbb{R}_{\geq 0} \rightarrow \mathbb{R}_{\geq 0}, \beta \in \mathcal{C}^{0}([0, \infty)), \varphi \in \mathcal{K}_{0}$, such that for every $(x, \lambda, t) \in(\overline{\mathcal{D}} \backslash\{0\}) \times \Lambda \times \mathbb{R}$ the following properties hold:

$$
\underline{\alpha}(\|x\|) \leq V(x) \leq \bar{\alpha}(\|x\|), \quad \frac{\partial V}{\partial x} f(x, \lambda, t) \leq \alpha(V(x))+\beta(V(x)) \varphi(|\lambda|) .
$$


Assumption 1 holds, for example, for systems in which the term $(\partial V / \partial x) f(x, \lambda, t)$ can be bounded from above as follows: there exist $\alpha_{0}, \beta_{0}, \varphi \in \mathcal{K}$ such that

(8) $\frac{\partial V}{\partial x} f(x, \lambda, t) \leq-\alpha_{0}(\|x\|)+\beta_{0}(\|x\|) \varphi(|\lambda|)$ for all $(x, \lambda, t) \in(\overline{\mathcal{D}} \backslash\{0\}) \times \Lambda \times \mathbb{R}$.

Indeed, (7) follows immediately from (8) with $\alpha(\cdot)=-\alpha_{0} \circ \bar{\alpha}^{-1}(\cdot), \beta(\cdot)=\beta_{0} \circ \underline{\alpha}^{-1}(\cdot)$, and $\underline{\alpha}^{-1}(\cdot), \bar{\alpha}^{-1}(\cdot)$ is the inverse of $\underline{\alpha}(\cdot), \bar{\alpha}(\cdot)$, respectively. In this case, Assumption 1 states that the zero solution of $(5 \bar{b})$ at $\lambda=0$ is globally asymptotically stable in the sense of Lyapunov, and $V(\cdot)$ is the corresponding Lyapunov function. Notice, however, that Lyapunov stability of the zero solution of (5b) at $\lambda=0$ is not needed for the assumption to hold. System (1b) is an example of a system in which the origin is unstable equilibrium, and yet Assumption 1 is satisfied with $V(x)=x^{2}, \mathcal{D}=$ $\left\{x \mid x \in \mathbb{R}_{>0}\right\}$ (see section 4.1 for more details). Finally, we remark that despite the right-hand side of (5a) being allowed to be time-varying, we restrict our consideration to systems for which the function $V(\cdot)$ does not depend on time explicitly.

Let us now proceed with detailing the requirements for the function $g(\cdot, \cdot, \cdot)$. These are presented in Assumption 2.

Assumption 2. There exist functions $\delta, \xi \in \mathcal{K}_{0}$ such that the following inequality holds for all $(x, \lambda, t) \in \mathcal{D}_{\Omega}$ :

$$
-\xi(|\lambda|)-\delta(\|x\|) \leq g(x, \lambda, t) \leq 0 .
$$

Assumption 2 reflects the fact that derivative $\dot{\lambda}$ does not change sign for all $(x, \lambda, t) \in \mathcal{D}_{\Omega}$. Without loss of generality we consider the case when $\lambda$ is nonincreasing with time. Alternative formulations of our conclusions for the case when (9) is replaced with $0 \leq g(x, \lambda, t) \leq \delta(\|x\|)+\xi(|\lambda|)$ are readily available. (In this case one may also need to redefine $\Lambda$ as an interval $\left[c_{1}, c_{2}\right], c_{1}<0, c_{2} \geq 0$.)

We aim to formulate a list of conditions that would allow us to estimate forward invariant sets of (5a) and, specifically, those in which the solutions of $(5 \mathrm{a})$ remain bounded. These conditions are provided in the next section.

3. Main results. Before providing formal statements of the results let us briefly comment on the internal structure of the section. We begin with section 3.1 presenting conditions for the existence of forward invariant sets for (5a) containing nontrivial bounded solutions in forward time. The conditions are constructive; i.e., not only is the existence of such sets guaranteed, but their boundaries are also explicitly provided. Two alternative statements of the results are discussed: one is limited to the case of differentiable boundaries (Lemma 1, section 3.1), and the other is applicable to nondifferentiable boundaries (Lemma 2, section 3.1). Estimates of the sets corresponding to solutions escaping the origin are provided in Lemmas 3 and 4 in section 3.2 .

3.1. Forward invariance. Our first result is provided in the lemma below.

Lemma 1 (boundedness 1). Let system (5a) be given and satisfying Assumptions 1 and 2. Suppose that the following holds:

(C1) there exist a function $\psi: \psi \in \mathcal{K} \cap \mathcal{C}^{1}((0, \infty))$ and $a \in \mathbb{R}_{>0}$ such that for all $V \in(0, a]$

$$
\frac{\partial \psi(V)}{\partial V}[\alpha(V)+\beta(V) \varphi(\psi(V))]+\delta\left(\underline{\alpha}^{-1}(V)\right)+\xi(\psi(V)) \leq 0,
$$

and suppose, in addition to $(\mathrm{C} 1)$, that the following hold: 

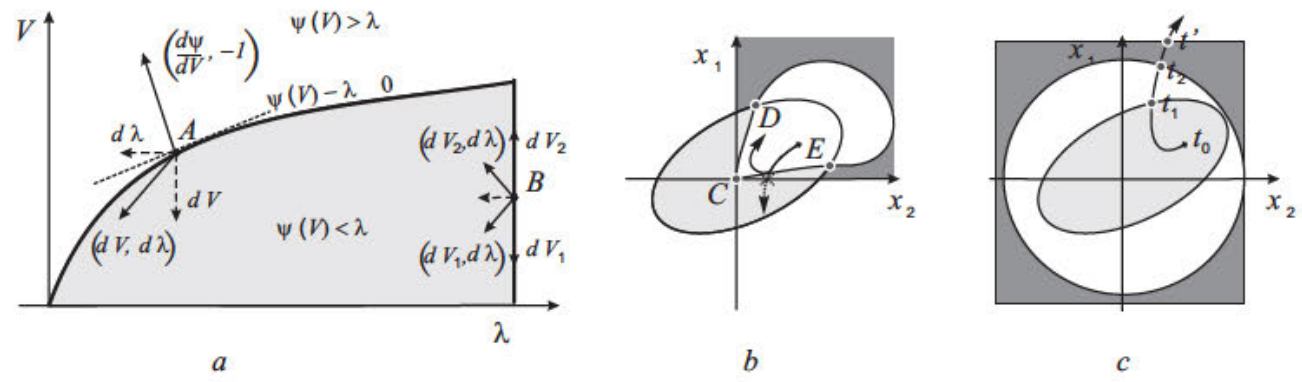

FIG. 2. Geometric interpretation of conditions of Lemma 1. Panel a: condition (C1). Panel b: condition (C2), first alternative. Panel c: condition (C2), second alternative. A more detailed explanation is provided in the text.

(C2) the set $\underline{\omega}(\psi(a))$ exists, and either the set $\overline{\mathcal{D}}$ contains $\underline{\omega}(\psi(a))$, or the ball $\left\{x \mid x \in \mathbb{R}^{n},\|x\| \leq \underline{\alpha}^{-1}(a)\right\}$ is in $\mathcal{D}$;

(C3) the set $\Omega_{a} \backslash\{(0,0)\}$, where

$$
\Omega_{a}=\left\{(x, \lambda) \mid x \in \underline{\omega}(\psi(a)), \lambda \in \mathbb{R}_{\geq 0}, \psi(a) \geq \lambda \geq \psi(V(x)), V(x) \in[0, a]\right\},
$$

is contained in the interior of $\overline{\mathcal{D}} \times \Lambda$.

Then the following holds:

(a) $\Omega_{a}$ is forward invariant with respect to (5a); that is, solutions of (5a) starting in $\Omega_{a}$ at $t=t_{0}$ are defined for all $t \geq t_{0}$, remain in $\Omega_{a}$, and are bounded.

Furthermore, for every solution of (5a) starting in $\Omega_{a}$, the following holds:

(b) There exists a limit

$$
\lim _{t \rightarrow \infty} \lambda(t)=\lambda^{\prime}, \quad \lambda^{\prime} \in[0, \psi(a)] .
$$

(c) If, in addition, the function $g(x, \lambda, \cdot)$ is uniformly continuous, then

$$
\lim _{t \rightarrow \infty} g\left(x(t), \lambda^{\prime}, t\right)=0 .
$$

Before providing a proof of the lemma let us first comment on its conditions. Condition (C1) is the actual criterion of forward invariance. Let us suppose, for simplicity, that $\overline{\mathcal{D}}$ coincides with $\mathbb{R}^{n}$, and conditions (C1)-(C3) are satisfied for some positive $a$. In this case, similar to the classical inequality in the method of Lyapunov functions for systems of ordinary differential equations $\dot{x}=F(x, t), F \in \mathcal{C}^{0}\left(\mathbb{R}^{n} \times \mathbb{R}\right)$ : $(\partial V / \partial x) F(x, t) \leq 0$, inequality (10) guarantees that all solutions of (5a) starting in (11) at $t=t_{0}$ exist for all $t \geq t_{0}$ and are bounded in forward time. Condition (C2) is a sort of domestication requirement. It is used in the proof to ensure that every solution of (5a) starting in $\Omega_{a}$ and leaving $\Omega_{a}$ through the boundary $\lambda=\psi(V(x))$ must necessarily contain a segment intersecting the boundary $\lambda=\psi(V(x))$ and lying entirely in $\mathcal{D} \times(0, \psi(a)]$. Condition (C3) is a technical requirement ensuring that every solution crossing through the boundary $\lambda=\psi(V(x)), \lambda \neq 0$ of $\Omega_{a}$ (if, of course, such a solution exists) at $t=t^{\prime}$ will remain in $\overline{\mathcal{D}} \times \Lambda$ over a nonempty interval $\left[t^{\prime}, t^{\prime \prime}\right]$, $t^{\prime \prime}>t^{\prime}$. A geometric interpretation of these conditions is provided in Figure 2.

Condition (C1) is illustrated in panel a. The vector $(\partial \psi / \partial V,-1)$ is normal to the curve $\lambda=\psi(V)$ at the point $A$. Since $\partial \psi / \partial V>0$, it is always pointing in the direction of $\lambda<\psi(V)$. One can easily see that (10) implies that for all $V \in(0, a]$ 
$\frac{\partial \psi}{\partial V} \dot{V}-\left.\dot{\lambda}\right|_{\lambda=\psi(V)} \leq 0$. Hence, according to $(10)$, the vector $(\dot{V}, \dot{\lambda})$ is pointing in the direction of $\lambda \geq \psi(V)$ on the surface $\lambda=\psi(V)$. Condition (C2) is illustrated in panels b and c. Panel b shows the case when the set $\overline{\mathcal{D}}$ contains $\underline{\omega}(\psi(a))$. Set $\mathcal{D}$ is the dark grey rectangle, the set $\lambda \geq \psi(V(x))$ for some fixed value of $\lambda$ is depicted as a light grey ellipse, and $\underline{\omega}(\psi(a))$ is the white area. The condition states that only those crossings through the boundary $\lambda=\psi(V(x))$ (segment DE in the figure) are allowed which occur in the white area. Solutions cannot cross segments CD and $\mathrm{CE}$ and hence must remain in $\overline{\mathcal{D}}$. Panel c concerns the second alternative, i.e., when the ball $\left\{x \mid x \in \mathbb{R}^{n},\|x\| \leq \underline{\alpha}^{-1}(a)\right\}$ is in $\mathcal{D}$. The white area depicts the ball $\left\{x \mid x \in \mathbb{R}^{n},\|x\| \leq \underline{\alpha}^{-1}(a)\right\}$. The ball contains sets $\left\{x \mid x \in \mathbb{R}^{n}, V(x) \leq c, c \in[0, a]\right\}$ as subsets. Given that the function $\psi(\cdot)$ is nondecreasing and strictly monotone, it is clear that the ball contains $\left\{x \mid x \in \mathbb{R}^{n}, \quad \lambda \geq \psi(V(x)), \lambda \in[0, \psi(a)]\right\}$. The condition therefore reflects that any solution $x\left(\cdot ; t_{0}, x_{0}, \lambda_{0}\right)$ leaving the set $\bar{D}$ at $t=t^{\prime}$ must necessarily cross through the surfaces $\lambda=\psi(V(x)), \lambda \in[0, \psi(a)]$, and $\|x\|=\underline{\alpha}^{-1}(a)$ at $t=t_{1}$ and $t=t_{2}, t_{2} \geq t_{1}$, respectively.

Proof. [Proof of Lemma 1] The proof of the lemma is split into two parts. In the first part we show that conditions (C2) and (C3) and the fact that Assumptions 1 and 2 hold guarantee that every solution of (5a) satisfying the initial condition $x\left(t_{0}\right)=x_{0}$, $\lambda\left(t_{0}\right)=\lambda_{0},\left(x_{0}, \lambda_{0}\right) \in \Omega_{a}$, will either (1) remain in $\underline{\omega}(\psi(a)) \times[0, \psi(a)]$ for $t \geq t_{0}$ (and consequently in $\overline{\mathcal{D}} \times[0, \psi(a)]$ ) as long as $\lambda\left(t ; t_{0}, x_{0}, \lambda_{0}\right) \geq 0$ (first alternative), or (2) if it leaves the set $\overline{\mathcal{D}} \times[0, \psi(a)]$ at some $t \geq t_{0}$, then it should first cross the boundary $\lambda=\psi(V(x)), \lambda \in(0, \psi(a)]$ (second alternative), in $\overline{\mathcal{D}} \times[0, \psi(a)]$. This ensures that inequalities (7), (9) in Assumptions 1 and 2 will hold along the solutions of (5a) starting in $\Omega_{a}$ at $t_{0}$ for $t \geq t_{0}$ as long as they remain in $\Omega_{a} \backslash\{(0,0)\}$. Furthermore, if the solution crosses through the boundary of the set $\Omega_{a} \backslash\{(0,0)\}$ at some $t \geq t_{0}$, then it must necessarily satisfy Assumptions 1 and 2 over a nonempty interval $\left[t, t^{\prime}\right]$, $t^{\prime}>t$, because the set $\Omega_{a} \backslash\{(0,0)\}$ is in the interior of $\overline{\mathcal{D}} \times \Lambda$.

In the second part of the proof we use this property to show that condition (10) is incompatible with the assertion that solutions of (5a) starting in $\Omega_{a}$ at $t=t_{0}$ may intersect the boundary $\lambda=\psi(V(x))$ at $t \geq t_{0}$.

Part 1. Let $\left(x_{0}, \lambda_{0}\right)$ be a point in $\Omega_{a}$. It is clear that solutions of (5a) exist at least locally and are unique. According to the first alternative of condition $(\mathrm{C} 2)$, that $\bar{D} \supset \underline{\omega}(\psi(a))$, components $x\left(t ; t_{0}, x_{0}, \lambda_{0}\right), x_{0} \in \underline{\omega}(\psi(a)), \lambda_{0} \in[0, \psi(a)]$ of the solutions of (5a) must belong to $\overline{\mathcal{D}}$ for $t \geq t_{0}$ as long as $\lambda\left(t ; t_{0}, x_{0}, \lambda_{0}\right) \geq 0$ (see Remark 1 ).

Consider the second alternative of $(\mathrm{C} 2) ; \mathcal{D}$ contains the ball $\left\{x \mid x \in \mathbb{R}^{n},\|x\| \leq\right.$ $\left.\underline{\alpha}^{-1}(a)\right\}$. Since the right-hand side of (5a) is locally Lipschitz, the equilibrium solution $x\left(\cdot ; t_{0}, 0,0\right) \equiv 0, \lambda\left(\cdot ; t_{0}, 0,0\right) \equiv 0$ is unique. Thus solutions $x\left(\cdot ; t_{0}, x_{0}, \lambda_{0}\right)$, $\lambda\left(\cdot ; t_{0}, x_{0}, \lambda_{0}\right),\left(x_{0}, \lambda_{0}\right) \in \Omega_{a}$ cannot escape the domain $\mathcal{D} \times[0, \psi(a)]$ through the point $(0,0)$. Let us show that if there is a solution $x\left(\cdot ; t_{0}, x_{0}, \lambda_{0}\right), \lambda\left(\cdot ; t_{0}, x_{0}, \lambda_{0}\right)$, $\left(x_{0}, \lambda_{0}\right) \in \Omega_{a}$ of $(5 \mathrm{a})$ that is leaving the set $\overline{\mathcal{D}} \times[0, \psi(a)]$ at some $t>t_{0}$, then it must first cross the boundary $\lambda=\psi(V(x)), \lambda \in(0, \psi(a)]$. Let this not be the case, and suppose that there exists a solution of (5a) touching the boundary of $\overline{\mathcal{D}}$ without crossing through $\lambda=\psi(V(x)), \lambda \in(0, \psi(a)]$. This means that there exists a $t^{\prime}>t_{0}$ such that

$$
\begin{aligned}
& \lambda\left(t^{\prime} ; t_{0}, x_{0}, \lambda_{0}\right) \geq \psi\left(V\left(x\left(t^{\prime} ; t_{0}, x_{0}, \lambda_{0}\right)\right),\right. \\
& \lambda\left(t^{\prime} ; t_{0}, x_{0}, \lambda_{0}\right) \in(0, \psi(a)]
\end{aligned}
$$

i.e., no crossing occurred, and yet the point $x\left(t^{\prime} ; t_{0}, x_{0}, \lambda_{0}\right)$ is on the boundary of $\overline{\mathcal{D}}$. It is therefore clear that the following must hold: $\psi\left(\underline{\alpha}\left(\left\|x\left(t^{\prime} ; t_{0}, x_{0}, \lambda_{0}\right)\right\|\right)\right)>\psi(a)$. On 
the other hand, according to Assumption 1 , we have that $\psi\left(\underline{\alpha}\left(\left\|x\left(t^{\prime} ; t_{0}, x_{0}, \lambda_{0}\right)\right\|\right)\right) \leq$ $\psi\left(V\left(x\left(t^{\prime} ; t_{0}, x_{0}, \lambda_{0}\right)\right)\right)$, and hence $\psi\left(V\left(x\left(t^{\prime} ; t_{0}, x_{0}, \lambda_{0}\right)\right)\right)>\psi(a)$. The latter inequality together with (14a) result in $\lambda\left(t^{\prime} ; t_{0}, x_{0}, \lambda_{0}\right)>\psi(a)$. This, however, contradicts (14b).

Part 2. We claim that any solution of (5a) passing through $\left(x_{0}, \lambda_{0}\right)$ at $t_{0}$ is defined for all $t \geq t_{0}$ and remains in $\Omega_{a}$ for all $t \geq t_{0}$. Let us first demonstrate that solutions of (5a) starting in $\Omega_{a} \backslash\{(0,0)\}$ cannot leave the set through the boundary $\lambda=\psi(V(x)), V(x) \in(0, a]$. Assume that this is not the case. Pick an arbitrary point $\left(x_{0}, \lambda_{0}\right) \in \Omega_{a} \backslash\{(0,0)\}$, let $\phi\left(\cdot ; t_{0}, x_{0}, \lambda_{0}\right)=\left(x\left(\cdot ; t_{0}, x_{0}, \lambda_{0}\right), \lambda\left(\cdot ; t_{0}, x_{0}, \lambda_{0}\right)\right),\left(x_{0}, \lambda_{0}\right) \in$ $\Omega_{a} \backslash\{(0,0)\}$, be the maximal solution of $(5 \mathrm{a})$, and let $\mathcal{T}=\left[t_{0}, t_{\max }\right)$ be the interval of its definition for $t>t_{0}$. Suppose that $\phi\left(\cdot ; t_{0}, x_{0}, \lambda_{0}\right)$ can cross through the boundary; i.e., there exists a $t^{\prime} \in \mathcal{T}$ such that $\lambda\left(t^{\prime} ; t_{0}, x_{0}, \lambda_{0}\right)<\psi\left(V\left(x\left(t^{\prime} ; t_{0}, x_{0}, \lambda_{0}\right)\right)\right)$. Condition (C3) states that $\Omega_{a}$ is in the interior of $\overline{\mathcal{D}} \times \Lambda$. Hence without loss of generality we can suppose that $\phi\left(t^{\prime} ; t_{0}, x_{0}, \lambda_{0}\right) \in \overline{\mathcal{D}} \times \Lambda$.

Consider the function $p: \mathcal{T} \rightarrow \mathbb{R}, p(t)=\psi\left(V\left(x\left(t ; t_{0}, x_{0}, \lambda_{0}\right)\right)\right)-\lambda\left(t ; t_{0}, x_{0}, \lambda_{0}\right)$. The function $p(\cdot)$ is continuous in $\mathcal{T}$. Thus there is a nonempty interval $\left[t_{1}, t^{\prime}\right] \subset \mathcal{T}$ such that $p\left(t_{1}\right)=0$ and $p(t)>0$ for all $t \in\left(t_{1}, t^{\prime}\right]$. Moreover, given that $\psi \in$ $\mathcal{C}^{1}((0, \infty))$, for every $t \in\left(t_{1}, t^{\prime}\right]$ the derivative $\dot{p}(t)$ exists, and $\dot{p}(\cdot)$ is locally bounded in $\mathcal{T}$. Therefore $p(\cdot)$ is absolutely continuous in $\left[t_{1}, t^{\prime}\right]$, and $p(t)=\int_{t_{1}}^{t} \dot{p}(\tau) d \tau>0, t \in$ $\left(t_{1}, t^{\prime}\right]$. According to the mean-value theorem there exists a $\tau \in\left(t_{1}, t\right]$ such that

$$
\begin{aligned}
p(t)= & \left(t-t_{1}\right) \dot{p}(\tau)=\left(t-t_{1}\right)\left[\frac{\partial \psi}{\partial V} \frac{\partial V}{\partial x} f\left(x\left(\tau ; t_{0}, x_{0}, \lambda_{0}\right), \lambda\left(\tau ; t_{0}, x_{0}, \lambda_{0}\right), \tau\right)\right. \\
& \left.-g\left(x\left(\tau ; t_{0}, x_{0}, \lambda_{0}\right), \lambda\left(\tau ; t_{0}, x_{0}, \lambda_{0}\right), \tau\right)\right] .
\end{aligned}
$$

Using the fact that the function $\psi(\cdot)$ is nondecreasing, i.e., $\partial \psi / \partial V \geq 0$ for all $V \in$ $(0, a]$, and invoking Assumptions 1 and 2 we derive that

$$
\begin{aligned}
\dot{p}(\tau) \leq & \frac{\partial \psi}{\partial V}\left[\alpha\left(V\left(x\left(\tau ; t_{0}, x_{0}, \lambda_{0}\right)\right)\right)+\beta\left(V\left(x\left(\tau ; t_{0}, x_{0}, \lambda_{0}\right)\right)\right) \varphi\left(\lambda\left(\tau ; t_{0}, x_{0}, \lambda_{0}\right)\right)\right] \\
& +\xi\left(\lambda\left(\tau ; t_{0}, x_{0}, \lambda_{0}\right)\right)+\delta\left(\left\|x\left(\tau ; t_{0}, x_{0}, \lambda_{0}\right)\right\|\right) .
\end{aligned}
$$

The functions $\xi(\cdot), \delta(\cdot), \varphi(\cdot)$ are nondecreasing, and

$$
\lambda\left(\tau ; t_{0}, x_{0}, \lambda_{0}\right)<\psi\left(V\left(x\left(\tau ; t_{0}, x_{0}, \lambda_{0}\right)\right)\right) .
$$

Hence invoking condition (C1) of the lemma, we can conclude that

$$
\begin{aligned}
\dot{p}(\tau) \leq & \frac{\partial \psi}{\partial V}\left[\alpha\left(V\left(x\left(\tau ; t_{0}, x_{0}, \lambda_{0}\right)\right)\right)+\beta\left(V\left(x\left(\tau ; t_{0}, x_{0}, \lambda_{0}\right)\right)\right) \varphi\left(\psi\left(V\left(x\left(\tau ; t_{0}, x_{0}, \lambda_{0}\right)\right)\right)\right)\right] \\
& +\xi\left(\psi\left(V\left(x\left(\tau ; t_{0}, x_{0}, \lambda_{0}\right)\right)\right)\right)+\delta\left(\underline{\alpha}^{-1}\left(V\left(x\left(\tau ; t_{0}, x_{0}, \lambda_{0}\right)\right)\right)\right) \leq 0 .
\end{aligned}
$$

On the other hand, since $p(t)>0$ and $\left(t-t_{1}\right)>0$ for all $t \in\left(t^{\prime}, t_{1}\right]$, the following must hold: $\dot{p}(\tau)>0$. This contradicts (15), and hence the statement that the solution $\phi\left(\cdot ; t_{0}, x_{0}, \lambda_{0}\right)$ crosses the boundary $\lambda=\psi(V(x))$ at some $t^{\prime} \in \mathcal{T}$ in $\overline{\mathcal{D}} \times \Lambda \backslash\{(0,0)\}$ in finite time is not true.

It is also clear that $\phi\left(\cdot ; t_{0}, x_{0}, \lambda_{0}\right)$ cannot escape $\Omega_{a}$ through the boundary $\lambda=$ $\psi(a)$ at any $t \in \mathcal{T}$ since the derivative $\dot{\lambda}(t)$ is nonpositive for all $t \in \mathcal{T}$. Finally, notice that the only remaining subset of the boundary of $\Omega_{a}$ through which the solutions may escape is the set $\left\{(x, \lambda) \mid x \in \mathbb{R}^{n}, \lambda \in \mathbb{R}, V(x)=0, \lambda=0\right\}$. This set, however, is the equilibrium of $(5 \mathrm{a})$, and the equilibrium solution $\phi\left(\cdot ; t_{0}, 0,0\right)=0$ is unique. 
Thus $\phi\left(t ; t_{0}, x_{0}, \lambda_{0}\right) \in \Omega_{a}$ for all $t \in \mathcal{T}$. Noticing that the set $\Omega_{a}$ is bounded and that the right-hand side of $(5 \mathrm{a})$ is locally Lipschitz, we conclude that $\phi\left(\cdot ; t_{0}, x_{0}, \lambda_{0}\right)$ is defined on $\left[t_{0}, \infty\right)$ and bounded. Given that $\left(x_{0}, \lambda_{0}\right)$ was an arbitrary point of $\Omega_{a} \backslash\{(0,0)\}$, that $t_{0}$ was chosen arbitrarily, and that the origin is the equilibrium of $(5 \mathrm{a})$, we conclude that all solutions of (5a) passing through $\Omega_{a}$ remain in $\Omega_{a}$ in forward time.

Property (12) is an immediate consequence of the Bolzano-Weierstrass theorem. In order to see that property (13) holds, we notice that the integral $\lambda\left(t ; t_{0}, x_{0}, \lambda_{0}\right)=$ $\lambda_{0}+\int_{t_{0}}^{t} g\left(x\left(\tau ; t_{0}, x_{0}, \lambda_{0}\right), \lambda\left(\tau ; t_{0}, x_{0}, \lambda_{0}\right), \tau\right) d \tau$ converges. Taking into account the boundedness of $x\left(\cdot ; t_{0}, x_{0}, \lambda_{0}\right), \lambda\left(\cdot ; t_{0}, x_{0}, \lambda_{0}\right)$ on $\left[t_{0}, \infty\right)$ and the fact that $g(\cdot, \cdot, t)$ is locally Lipschitz uniformly in $t$ and is uniformly continuous in $t$, we conclude that the function $g\left(x\left(\cdot ; t_{0}, x_{0}, \lambda_{0}\right), \lambda\left(\cdot ; t_{0}, x_{0}, \lambda_{0}\right), \cdot\right)$ is uniformly continuous on $\left[t_{0}, \infty\right)$. Thus, according to Barbalat's lemma (see, e.g., [7]),

$$
\lim _{t \rightarrow \infty} g\left(x\left(t ; t_{0}, x_{0}, \lambda_{0}\right), \lambda\left(t ; t_{0}, x_{0}, \lambda_{0}\right), t\right)=0 .
$$

Hence the result follows as a consequence of (12) and from the continuity of $g(x, \cdot, t)$ in $\Lambda$.

Remark 2. Notice that if the interior of $\underline{\omega}(\psi(a))$ is not empty and its closure contains the origin, then the measure of $\Omega_{a}, a>0$, is not zero. Indeed, since the function $\psi(\cdot)$ is strictly monotone, $\psi(a)>0$. Pick a $\lambda^{\prime} \in(0, \psi(a))$, and let $\mathcal{B}(x, r)$ denote an open ball of radius $r$ in $\mathbb{R}^{n}$ centered at $x$. The ball $\mathcal{B}\left(0,\left(\bar{\alpha}^{-1} \circ \psi^{-1}\right)\left(\lambda^{\prime}\right)\right)$ is contained in the set $\left\{x \in \mathbb{R}^{n} \mid x: \psi^{-1}\left(\lambda^{\prime}\right)>V(x)\right\}$. Since the closure of $\underline{\omega}(\psi(a))$ contains the origin and the interior of $\underline{\omega}(\psi(a))$ is open, we can conclude that there exists a ball $\mathcal{B}\left(x^{\prime}, r\right)$ such that $\mathcal{B}\left(x^{\prime}, r\right) \subset \underline{\omega}(\psi(a))$ and $\mathcal{B}\left(x^{\prime}, r\right) \subset \mathcal{B}\left(0,\left(\bar{\alpha}^{-1} \circ \psi^{-1}\right)\left(\lambda^{\prime}\right)\right)$. Thus the set $\mathcal{B}\left(x^{\prime}, r\right) \times\left\{\lambda^{\prime}\right\}$ is in the interior of $\Omega_{a}$. It is also clear that the set $\overline{\mathcal{B}}\left(x^{\prime}, r\right) \times\left[\lambda^{\prime}, \lambda^{\prime \prime}\right], \psi(a)>\lambda^{\prime \prime}>\lambda^{\prime}$ is in the interior of $\Omega_{a}$ provided that $\lambda^{\prime \prime}$ is sufficiently close to $\lambda^{\prime}$. Thus the measure of $\Omega_{a}$ is not zero.

Remark 3. According to the assumptions of Lemma $1, d \psi\left(V\left(x\left(t ; x_{0}, \lambda_{0}\right)\right)\right) / d t$ is nonpositive in the set $\left\{(\lambda, x) \lambda \in \mathbb{R}_{>0}, x \in \mathbb{R}^{n} \mid \lambda=\psi(V(x)), V(x) \in[0, a]\right\}$. Hence if $V(x) \in[0, a]$ and $t \geq t_{0}$, then

$$
\frac{\partial V}{\partial x} f(x, \psi(V(x)), t) \leq 0 .
$$

Therefore, subject to the assumptions of the lemma, (16) may be used as a necessary condition for testing positive invariance of the sets $\Omega_{a}$.

Remark 4. If the second alternative of $(\mathrm{C} 2)$ holds, then the requirement that $\underline{\omega}(\psi(a))$ exists is not necessary, and the definition of $\Omega_{a}$ may be replaced with $\Omega_{a}=$ $\left\{(x, \lambda) \mid x \in \mathbb{R}^{n}, \lambda \in \mathbb{R}_{\geq 0}, \psi(a) \geq \lambda \geq \psi(V(x)), V(x) \in[0, a]\right\}$.

Notice also that if $\mathcal{D}, \Lambda$ coincide with $\mathbb{R}^{n}$ and $\mathbb{R}$, respectively, then Lemma 1 reduces to the much simpler statement below.

Corollary 1. Consider system (5a), and let $\mathcal{D}=\mathbb{R}^{n}, \Lambda=\mathbb{R}$. Suppose that Assumptions 1 and 2 hold and there exist a function $\psi: \psi \in \mathcal{K} \cap \mathcal{C}^{1}((0, \infty))$ and a positive constant a such that (10) holds. Then the set

$$
\Omega_{a}=\left\{(x, \lambda) \mid x \in \mathbb{R}^{n}, \lambda \in \mathbb{R}_{\geq 0}, \psi(a) \geq \lambda \geq \psi(V(x)), V(x) \in[0, a]\right\}
$$

is forward invariant, and conclusions (b), (c) of Lemma 1 hold.

Lemma 1 requires that the function $\psi(\cdot)$ used in the definition of the set $\Omega_{a}$ be strictly increasing and differentiable. Occasionally, a need might arise for functions 
$\psi(\cdot)$ which are not differentiable or not strictly monotone. While the requirement of strict monotonicity can be traded for the weaker constraint that the function $\psi(\cdot)$ be nondecreasing, without significant alternations to the statements of Lemma 1 and Corollary 1 and their proofs, dealing with the issue of differentiability involves replacing (10) with a set of different invariance conditions. In what follows we present these modified conditions involving the notion of a star-shaped set. A formal definition and the basic properties of star-shaped sets and functions are provided in the appendix. A brief summary of notions that are essential for the formulation of the results is provided below.

Recall that a set $\Omega \subset \mathbb{R}^{n}$ is called star-shaped w.r.t. the origin if every segment connecting the origin with a point $p \in \Omega$ lies entirely in $\Omega$. Clearly, convexity of a set containing the origin implies that it is also star-shaped w.r.t. the origin (since a convex set is star-shaped w.r.t. every point of that set). The star-shaped envelope of a set $D$ (w.r.t. the origin) is the minimal star-shaped set (w.r.t. the origin) including $D$; that is, every star-shaped set (w.r.t. the origin) including $D$ as a subset must necessarily include the star-shaped envelope (w.r.t. the origin) of $D$ as well. This set is denoted as $\operatorname{star}(D)$. (In the appendix, for a star shaped envelope of a set w.r.t. a point $x$, the notation $\operatorname{star}_{x}(D)$ is used. For notational convenience we omit the subscript 0 when referring to star-shaped sets, envelopes, and functions w.r.t. the origin.) The epigraph, respectively, hypograph, of a function $f: \mathbb{R}^{n} \rightarrow \mathbb{R}$, or simply $\operatorname{epi}(f)$, is the set in $\mathbb{R}^{n+1}$ : $\operatorname{epi}(f)=\left\{(x, \mu) \mid x \in \mathbb{R}^{n}, \mu \in \mathbb{R}, f(x) \leq \mu\right\}$, respectively, $\operatorname{hyp}(f)=\left\{(x, \mu) \mid x \in \mathbb{R}^{n}, \mu \in \mathbb{R}, f(x) \geq \mu\right\}$. A function $f: \mathbb{R}^{n} \rightarrow \mathbb{R}$ is called star-shaped w.r.t. the origin iff its epigraph is a star-shaped set w.r.t. the origin. The convex envelope of a function $f: \mathbb{R}^{n} \rightarrow \mathbb{R}$ is denoted as $\operatorname{conv}(f)(x)$, and the starshaped envelope of $f(\cdot)$ (w.r.t. the origin) is denoted as $\operatorname{star}(f)(x)$. Let $f: \mathbb{R} \rightarrow \mathbb{R}$, and let $[0, a], a>0$, be an interval. We define

$$
\begin{aligned}
\operatorname{epi}\left(f_{[0, a]}\right) & =\{(x, \mu) \mid x \in[0, a], \mu \in \mathbb{R}, f(x) \leq \mu\}, \\
\operatorname{hyp}\left(f_{[0, a]}\right) & =\{(x, \mu) \mid x \in[0, a], \mu \in \mathbb{R}, f(x) \geq \mu\}
\end{aligned}
$$

as the epigraph and hypograph of the restriction of $f$ on the interval $[0, a]$. Now we are ready to formulate the following.

Lemma 2 (boundedness 2). Let system (5a) be given, and let it satisfy Assumptions 1 and 2. Suppose that the following holds:

(C4) There exists a function $\psi \in \mathcal{K}$ such that for some $a \in \mathbb{R}_{>0}$ the set epi $\left(\psi_{[0, a]}\right)$ is star-shaped w.r.t. the origin, and for all $V \in[0, a]$

$$
\psi(V)[\alpha(V)+\beta(V) \varphi(\psi(V))]+V\left[\delta\left(\underline{\alpha}^{-1}(V)\right)+\xi(\psi(V))\right] \leq 0 .
$$

Furthermore, let conditions (C2) and (C3) of Lemma 1 hold.

Then $\Omega_{a}$ is forward invariant w.r.t. (5a), and, moreover, conclusions (b), (c) of Lemma 1 apply.

Proof of Lemma 2. The proof is similar to that of Lemma 1.

As has already been shown, conditions (C2) and (C3) and Assumptions 1 and 2 imply that if the solution $x\left(\cdot ; t_{0}, x_{0}, \lambda_{0}\right), \lambda\left(\cdot ; t_{0}, x_{0}, \lambda_{0}\right),\left(x_{0}, \lambda_{0}\right) \in \Omega_{a}$, leaves the set $\Omega_{a}$ at some $t \geq t_{0}$, then it must necessarily satisfy Assumptions 1 and 2 over a nonempty interval $\left[t, t^{\prime}\right], t^{\prime}>t$. We will show now that condition (18) is incompatible with the claim that solutions of the system starting in the set $\Omega_{a}$ at $t=t_{0}$ may leave the set at some $t^{\prime} \geq t_{0}$.

Let $\left(x_{0}, \lambda_{0}\right) \in \Omega_{a}$, and let $\phi\left(\cdot ; t_{0}, x_{0}, \lambda_{0}\right)$ be a solution of $(5 \mathrm{a})$. The solution exists at least locally; let $\mathcal{T}=\left[t_{0}, t_{\max }\right)$ be its maximal interval of definition for 
$t \geq t_{0}$. First, we show that if $\left(x_{0}, \lambda_{0}\right) \neq(0,0)$, then the solution $\phi\left(\cdot ; t_{0}, x_{0}, \lambda_{0}\right)$ does not leave $\Omega_{a}$ through the boundary $\lambda=\psi(V(x)), V(x) \in(0, a]$, at any $t \in \mathcal{T}$. Suppose that this is not the case, and there exists a $t^{\prime} \in \mathcal{T}$ such that $\lambda\left(t^{\prime} ; t_{0}, x_{0}, \lambda_{0}\right)<$ $\psi\left(V\left(x\left(t^{\prime} ; t_{0}, x_{0}, \lambda_{0}\right)\right)\right)$. Without loss of generality we can assume that the value of $t^{\prime}$ is such that $\lambda\left(t ; t_{0}, x_{0}, \lambda_{0}\right) \geq \psi\left(V\left(x\left(t ; t_{0}, x_{0}, \lambda_{0}\right)\right)\right)$ for all $t \in\left[t_{0}, t_{1}\right], t_{0} \leq t_{1}$, but $\lambda\left(t ; t_{0}, x_{0}, \lambda_{0}\right)<\psi\left(V\left(x\left(t ; t_{0}, x_{0}, \lambda_{0}\right)\right)\right)$ for $t \in\left(t_{1}, t^{\prime}\right]$. Further, let $t^{\prime}$ be close enough to $t_{0}$ so that $\lambda\left(t ; t_{0}, x_{0}, \lambda_{0}\right) \neq 0$ for all $t \in\left[t_{0}, t^{\prime}\right]$. It is clear that making such a choice of $t^{\prime}$ is always possible because the value of $\lambda\left(t_{1} ; t_{0}, x_{0}, \lambda_{0}\right)$ must necessarily be positive, and that $\lambda\left(\cdot ; t_{0}, x_{0}, \lambda_{0}\right)$ is continuous on $\mathcal{T}$.

Introduce the function $p:\left[t_{0}, t^{\prime}\right] \rightarrow \mathbb{R}: p(t)=V\left(x\left(t ; t_{0}, x_{0}, \lambda_{0}\right)\right) / \lambda\left(t ; t_{0}, x_{0}, \lambda_{0}\right)$. The function $p(\cdot)$ is defined on $\left[t_{0}, t^{\prime}\right]$, and, moreover, it is continuous and is continuously differentiable on $\left[t_{0}, t^{\prime}\right]$. Thus $p(\cdot)$ is absolutely continuous on $\left[t_{0}, t^{\prime}\right]$, and $p(t)=$ $p\left(t_{1}\right)+\int_{t_{1}}^{t} \dot{p}(\tau) d \tau$. Notice that $p(t)>p\left(t_{1}\right)$ for $t \in\left(t_{1}, t^{\prime}\right]$. Indeed, if $p(t) \leq p\left(t_{1}\right)$, then the point $\left(V\left(x\left(t ; t_{0}, x_{0}, \lambda_{0}\right)\right), \lambda\left(t ; t_{0}, x_{0}, \lambda_{0}\right)\right)$ would belong to the set epi $\left(\psi_{[0, a]}\right)$, which contradicts the earlier established property that $\left.\psi\left(V\left(x\left(t ; t_{0}, x_{0}, \lambda_{0}\right)\right)\right)<\lambda\left(t ; t_{0}, x_{0}, \lambda_{0}\right)\right)$ for all $t \in\left(t_{1}, t^{\prime}\right]$.

In order to see this consider the point $\left(V\left(x\left(t_{1} ; t_{0}, x_{0}, \lambda_{0}\right)\right), \lambda\left(t_{1} ; t_{0}, x_{0}, \lambda_{0}\right)\right)$. According to the choice of $t_{1}$, this point is from epi $\left(\psi_{[0, a]}\right)$. Given that epi $\left(\psi_{[0, a]}\right)$ is star-shaped (condition (C4) of the lemma), every pair $(v, \lambda): \lambda \geq v / p\left(t_{1}\right), v \in$ $\left[0, V\left(x\left(t_{1} ; t_{0}, x_{0}, \lambda_{0}\right)\right]\right.$, belongs to epi $\left(\psi_{[0, a]}\right)$. Noticing that

$$
\lambda\left(t ; t_{0}, x_{0}, \lambda_{0}\right) \leq \lambda\left(t_{1} ; t_{0}, x_{0}, \lambda_{0}\right) \text { for all } t \in\left(t_{1}, t^{\prime}\right]
$$

we conclude that the condition $p(t) \leq p\left(t_{1}\right)$ for all $t \geq t_{1}$ implies that $V\left(x\left(t ; t_{0}, x_{0}, \lambda_{0}\right)\right)$ $\leq V\left(x\left(t_{1} ; t_{0}, x_{0}, \lambda_{0}\right)\right)$ for all $t \in\left(t_{1}, t^{\prime}\right]$. Thus points $\left(V\left(x\left(t ; t_{0}, x_{0}, \lambda_{0}\right)\right), \lambda\right)$ such that $\lambda \geq V\left(x\left(t ; t_{0}, x_{0}, \lambda_{0}\right)\right) / p\left(t_{1}\right)$ are in epi $\left(\psi_{[0, a]}\right)$. This includes points $\left(V\left(x\left(t ; t_{0}, x_{0}, \lambda_{0}\right)\right.\right.$, $\left.\lambda\left(t ; t_{0}, x_{0}, \lambda_{0}\right)\right)$ for $t \in\left(t_{1}, t^{\prime}\right]$ provided that $p(t)=V\left(x\left(t ; t_{0}, x_{0}, \lambda_{0}\right)\right) / \lambda\left(t ; t_{0}, x_{0}, \lambda_{0}\right) \leq$ $p\left(t_{1}\right)$ for $t \in\left(t_{1}, t^{\prime}\right]$.

Having derived that $p(t)>p\left(t_{1}\right)$ for all $t \in\left(t_{1}, t^{\prime}\right]$ we therefore obtain $\int_{t_{1}}^{t^{\prime}} \dot{p}(s) d s>$ 0 . According to the mean-value theorem, there is a $\tau \in\left(t_{1}, t^{\prime}\right]$ such that $\dot{p}(\tau)>0$. Assumptions 1 and 2, however, imply that

$$
\dot{p}(\tau)=\frac{\dot{V}\left(x\left(\tau ; t_{0}, x_{0}, \lambda_{0}\right)\right) \lambda\left(\tau ; t_{0}, x_{0}, \lambda_{0}\right)-\dot{\lambda}\left(\tau ; t_{0}, x_{0}, \lambda_{0}\right) V\left(x\left(\tau ; t_{0}, x_{0}, \lambda_{0}\right)\right)}{\lambda^{2}\left(\tau ; t_{0}, x_{0}, \lambda_{0}\right)} \leq 0
$$

because condition $(\mathrm{C} 4)$ of the lemma demands that

$$
\begin{aligned}
\dot{V}(x & \left.\left(\tau ; t_{0}, x_{0}, \lambda_{0}\right)\right) \lambda\left(\tau ; t_{0}, x_{0}, \lambda_{0}\right)-\dot{\lambda}\left(\tau ; t_{0}, x_{0}, \lambda_{0}\right) V\left(x\left(\tau ; t_{0}, x_{0}, \lambda_{0}\right)\right) \\
\leq & \psi\left(V\left(x\left(\tau ; t_{0}, x_{0}, \lambda_{0}\right)\right)\right)\left[\alpha\left(V\left(x ; t_{0}, x_{0}, \lambda_{0}\right)\right)\right) \\
& \left.+\beta\left(V\left(x\left(\tau ; t_{0}, x_{0}, \lambda_{0}\right)\right)\right) \varphi\left(\psi\left(V\left(x\left(\tau ; t_{0}, x_{0}, \lambda_{0}\right)\right)\right)\right)\right] \\
& +V\left[\delta\left(\underline{\alpha}^{-1}\left(V\left(x\left(\tau ; t_{0}, x_{0}, \lambda_{0}\right)\right)\right)\right)+\xi\left(\psi\left(V\left(x\left(\tau ; t_{0}, x_{0}, \lambda_{0}\right)\right)\right)\right)\right] \leq 0 .
\end{aligned}
$$

This is clearly a contradiction, and hence the solution $\phi\left(\cdot ; t_{0}, x_{0}, \lambda_{0}\right)$ does not cross the boundary $\lambda=\psi(V(x)), V \in(0, a]$, of $\Omega_{a}$ at any $t \in \mathcal{T}$. The rest of the proof is identical to that of Lemma 1.

There is a simple geometric interpretation of the conditions of Lemma 2 (see Figure 3). Consider an interval $(0, a]$ such that the epigraph of $\psi$ for $V \in[0, a]$ : $\operatorname{epi}\left(\psi_{[0, a]}\right)=\left\{(V, \lambda) \mid V \in[0, a], \lambda \in \mathbb{R}_{\geq 0}, \psi(V)<\lambda\right\}$ is star-shaped w.r.t. the origin. It is clear that if the vector $(\dot{V}, \dot{\lambda})$ at the boundary $\lambda=\psi(V), V \in(0, a]$, 


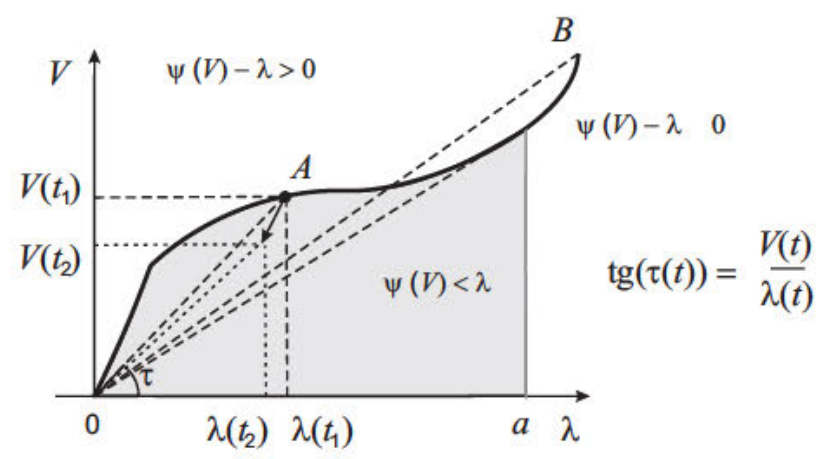

FIG. 3. Conditions of forward invariance with nondifferentiable function $\psi(\cdot)$. Star-shapedness of the epi $(\psi)$ is needed to guarantee that the vector $\left(V\left(t_{1}\right)-V\left(t_{2}\right), \lambda\left(t_{1}\right)-\lambda\left(t_{2}\right)\right)$ points in the direction of $\psi(V)<\lambda$ for all points on the curve $\lambda=\psi(V), V \in[0, a]$. Notice that this condition does not hold for the point $B$.

is always pointing inside epi $\left(\psi_{[0, a]}\right)$, then $V\left(x\left(t ; t_{0}, x_{0}, \lambda_{0}\right)\right), \lambda\left(t ; x_{0}, \lambda_{0}\right),\left(x_{0}, \lambda_{0} \in \Omega_{a}\right)$ will remain in $\Omega_{a}$ for all $t \geq t_{0}$. A sufficient condition for the latter, as is illustrated in Figure 3, is that the ratio $p(t)=V\left(x\left(t ; t_{0}, x_{0}, \lambda_{0}\right)\right) / \lambda\left(t ; t_{0}, x_{0}, \lambda_{0}\right)$ is nonincreasing with $t, t \geq t_{0}$, for all $V \in[0, a], \lambda=\psi(V)$. Rewriting this requirement as $\dot{p}(t) \leq 0$ for all $t \geq t_{0}$ and invoking the estimates in Assumptions 1 and 2 results in (18).

Remark 5. Note that Lemmas 1 and 2 and Corollary 1 can be straightforwardly generalized to account for a wider range of systems (5a). In particular, in Assumption 1 , instead of requiring the existence of $V: \mathbb{R}^{n} \rightarrow \mathbb{R}$ such that (7) holds, one can require that a weaker condition be satisfied: There exist a function of $n+1$ real variables, $V: \mathbb{R}^{n} \times \mathbb{R} \rightarrow \mathbb{R}$, and two functions of one variable, $\underline{\alpha}, \bar{\alpha} \in \mathcal{K}_{\infty}$, such that $V(\cdot, \cdot)$ is continuously differentiable except (possibly) on the ray $\left\{(x, t) \mid x \in \mathbb{R}^{n}\right.$, $\left.t \in \mathbb{R}, t \geq t_{0}, x=0\right\}$, and

$$
\underline{\alpha}(\|x\|) \leq V(x, t) \leq \bar{\alpha}(\|x\|), \quad \frac{\partial V}{\partial x} f(x, \lambda, t)+\frac{\partial V}{\partial t} \leq \alpha(V(x, t))+\beta(V(x, t)) \varphi(|\lambda|) .
$$

It is clear that statements of the results presented so far will remain the same, and also that their proofs will be almost identical under such a modification. The only visible differences would be notational, making technical derivations look more complicated.

3.2. Solutions escaping a neighborhood of the origin. In the previous sections we focused predominantly on developing boundedness and convergence tests for the solutions of $(5 \mathrm{a})$. It may sometimes be desirable to consider a complementary problem: determine whether a solution passing through some given point escapes the vicinity of the origin. As we show below, the very same set of techniques that was employed in the proofs of Lemmas 1 and 2 can be applied for solving the complementary problem as well.

Naive intuition suggests that criteria specifying domains of initial conditions corresponding to the solutions escaping small neighborhoods of the origin could be in a dual relation to the convergence and boundedness tests we formulated earlier. In some sense, this is indeed the case. Consider the assumptions of Lemmas 1 and 2. In the proofs of these lemmas, conditions (7), (9) played crucial role. These conditions provided estimates of the upper bounds for $V,-\lambda$ as functions of $V$ and $\lambda$. These upper bounds are used in conditions (10), (18), which are shown to be incompatible 
with the assumption that the solutions passing through the interior of $\Omega_{a}$ can cross the boundary $\lambda=\psi(V(x))$. Thus one may expect that a dual result might involve a set of conditions which is dual to (7), (9). For this purpose we consider the following substitutes for Assumptions 1 and 2.

Assumption 3. There exist a continuous function $V: \mathbb{R}^{n} \rightarrow \mathbb{R}$, differentiable everywhere except possibly at the origin, and five functions of one variable, $\underline{\alpha}, \bar{\alpha} \in \mathcal{K}_{\infty}$, $\alpha: \mathbb{R}_{\geq 0} \rightarrow \mathbb{R}, \alpha(0)=0, \beta: \mathbb{R}_{\geq 0} \rightarrow \mathbb{R}_{\geq 0}, \beta \in \mathcal{C}^{0}([0, \infty)), \varphi \in \mathcal{K}_{0}$, such that for every $(x, \lambda, t) \in \mathcal{D}_{\Omega}$ the following properties hold:

$$
\underline{\alpha}(\|x\|) \leq V(x) \leq \bar{\alpha}(\|x\|), \quad \frac{\partial V}{\partial x} f(x, \lambda, t) \geq \alpha(V(x))-\beta(V(x)) \varphi(|\lambda|) .
$$

Assumption 3 is similar to Assumption 1, except for the sign of the last inequality and also for the sign with which the term $\beta(V) \varphi(|\lambda|)$ enters the right-hand side of (20). In essence, it states that one can provide a lower bound for the derivative of $V$ as a function of $V$ and $\lambda$. Let us proceed with determining a substitute for Assumption 2.

Assumption 4 . There exists a function $\delta \in \mathcal{K}_{0}$ such that the following inequality holds for all $(x, \lambda, t) \in \mathcal{D}_{\Omega}$ :

$$
g(x, \lambda, t) \leq-\delta(\|x\|)
$$

Similarly to the case of Assumptions 1 and 3, Assumption 4 is almost a copy of Assumption 2, in which the sign of the inequality is reversed. The other difference is that we suppose that there is an upper bound for $g(\cdot, \cdot, \cdot)$, and this bound is independent of $\lambda$. It is clear, however, that if such a dependence were to be in the form $g(x, \lambda, t) \leq-\delta(\|x\|)-\xi(|\lambda|), \delta, \xi \in \mathcal{K}_{0}$, then it could be reduced to that stated in (21).

LEMma 3 (solutions escaping a neighborhood of the origin). Let system (5a) be given and satisfying Assumptions 3 and 4 . Suppose that $\mathcal{D}$ contains the origin. In addition, let the following hold:

(C5) there exist a function $\psi: \psi \in \mathcal{K}_{\infty} \cap \mathcal{C}^{1}((0, \infty))$ and an $a \in \mathbb{R}_{>0}$ such that for some $\varepsilon \in \mathbb{R}_{>0}$ and all $V \in(0, a]$ the following holds:

$$
\frac{\partial \psi(V)}{\partial V}[\alpha(V)-\beta(V) \varphi(\psi(V)-\varepsilon)]+\delta\left(\bar{\alpha}^{-1}(V)\right) \geq 0 ;
$$

(C6) the set $\Omega_{a}$, where

$$
\Omega_{a}=\{(x, \lambda) \mid x \in \overline{\mathcal{D}}, \psi(a)-\varepsilon \geq \lambda \geq \psi(V(x))-\varepsilon, V(x) \in[0, a]\},
$$

is contained in $\mathcal{D} \times\left(c_{1}, c_{2}\right)$.

Let $\Omega_{a}^{*}$ be the complement of $\Omega_{a}$ in $\overline{\mathcal{D}} \times\left[c_{1}, \psi(a)-\varepsilon\right]$. Then solutions of (5a) starting in $\Omega_{a}^{*}$ at $t=t_{0}$ cannot converge to the origin without leaving the set $\overline{\mathcal{D}} \times$ $\left[c_{1}, \psi(a)-\varepsilon\right]$ at least once.

Proof of Lemma 3 . Let $\phi\left(\cdot ; t_{0}, x_{0}, \lambda_{0}\right)=\left(x\left(\cdot ; t_{0}, x_{0}, \lambda_{0}\right), \lambda\left(\cdot ; t_{0}, x_{0}, \lambda_{0}\right)\right),\left(x_{0}, \lambda_{0}\right) \in$ $\Omega_{a}^{*}$, be a solution of (5a) converging to the origin as in forward time without leaving the set $\overline{\mathcal{D}} \times\left[c_{1}, \psi(a)-\varepsilon\right]$. This implies that for an $r>0$ (sufficiently small) there is a $t_{1}>t_{0}$ such that $\lambda^{2}\left(t_{1} ; t_{0}, x_{0}, \lambda_{0}\right)+\left\|x\left(t_{1} ; t_{0}, x_{0}, \lambda_{0}\right)\right\|^{2}=r^{2}$, and that $\phi\left(t ; t_{0}, x_{0}, \lambda_{0}\right) \in$ $\overline{\mathcal{D}} \times\left[c_{1}, \psi(a)-\varepsilon\right]$ for all $t \geq t_{0}$. Let us pick $r=\left(\bar{\alpha}^{-1} \circ \psi^{-1}\right)(\varepsilon) / 2$. Notice that $\lambda\left(t_{1} ; t_{0}, x_{0}, \lambda_{0}\right)$ is positive since $\dot{\lambda}=g(x, \lambda, t)$ is nonpositive in $\mathcal{D}_{\Omega}$. Thus the following 
estimate holds:

$$
\begin{aligned}
\left\|x\left(t_{1} ; t_{0}, x_{0}, \lambda_{0}\right)\right\| & =\sqrt{r^{2}-\lambda\left(t_{1} ; t_{0}, x_{0}, \lambda_{0}\right)}<\left(\bar{\alpha}^{-1} \circ \psi^{-1}\right)(\varepsilon) \\
& <\left(\bar{\alpha}^{-1} \circ \psi^{-1}\right)\left(\varepsilon+\lambda\left(t_{1} ; t_{0}, x_{0}, \lambda_{0}\right)\right) .
\end{aligned}
$$

This, however, implies that $\psi\left(\bar{\alpha}\left(\left\|x\left(t_{1} ; t_{0}, x_{0}, \lambda_{0}\right)\right\|\right)\right)<\lambda\left(t_{1} ; t_{0}, x_{0}, \lambda_{0}\right)+\varepsilon$, and subsequently that $\lambda\left(t_{1} ; t_{0}, x_{0}, \lambda_{0}\right)>\psi\left(V\left(x\left(t_{1} ; t_{0}, x_{0}, \lambda_{0}\right)\right)\right)-\varepsilon$.

Introduce the function $p:\left[t_{0}, \infty\right) \rightarrow \mathbb{R}:$

$$
p(t)=\psi\left(V\left(x\left(t ; t_{0}, x_{0}, \lambda_{0}\right)\right)\right)-\lambda\left(t ; t_{0}, x_{0}, \lambda_{0}\right)-\varepsilon .
$$

The function $p(\cdot)$ is continuous and differentiable on $\left[t_{0}, t_{1}\right]$, and $\dot{p}(\cdot)$ is clearly bounded. Since $p\left(t_{0}\right)>0$ (condition $(\mathrm{C} 6)$ ) and $p\left(t_{1}\right)<0$ then, according to the intermediate value theorem, there must be a point $t^{\prime} \in\left(t_{0}, t_{1}\right)$ such that $p\left(t^{\prime}\right)=0$. Without loss of generality we can suppose that $p(t)>0$ for all $t \in\left[t_{0}, t^{\prime}\right)$. Let $t^{\prime \prime}$ be a point in $\left(t_{0}, t^{\prime}\right)$; then

$$
p\left(t^{\prime}\right)-p\left(t^{\prime \prime}\right)=\int_{t^{\prime \prime}}^{t^{\prime}} \dot{p}(s) d s=\left(t^{\prime}-t^{\prime \prime}\right)\left(\frac{\partial \psi}{\partial V} \dot{V}(\tau)-\dot{\lambda}(\tau)\right)<0
$$

where $\tau$ belongs to $\left[t^{\prime \prime}, t^{\prime}\right]$. Recall that we assumed that $\phi\left(t ; t_{0}, x_{0}, \lambda_{0}\right) \in \overline{\mathcal{D}} \times\left[c_{1}, \psi(a)-\right.$ $\varepsilon]$ for all $t \geq t_{0}$, including for $t \in\left[t^{\prime \prime}, t^{\prime}\right]$. According to Assumptions 3 and 4 , the fact that $\lambda\left(\tau ; t_{0}, x_{0}, \lambda_{0}\right) \leq \psi\left(V\left(x\left(\tau ; t_{0}, x_{0}, \lambda_{0}\right)\right)\right)-\varepsilon$, and condition (C5), the following should hold:

$$
\begin{aligned}
& \frac{\partial \psi}{\partial V} \dot{V}(\tau)-\dot{\lambda}(\tau) \geq \frac{\partial \psi}{\partial V}\left[\alpha\left(V\left(x\left(\tau ; t_{0}, x_{0}, \lambda_{0}\right)\right)\right)\right. \\
& \left.\quad-\beta\left(V\left(x\left(\tau ; t_{0}, x_{0}, \lambda_{0}\right)\right)\right) \varphi\left(\left|\lambda\left(\tau ; t_{0}, x_{0}, \lambda_{0}\right)\right|\right)\right]+\delta\left(\left\|x\left(\tau ; t_{0}, x_{0}, \lambda_{0}\right)\right\|\right) \\
& \geq \\
& \quad \frac{\partial \psi}{\partial V}\left[\alpha\left(V\left(x\left(\tau ; t_{0}, x_{0}, \lambda_{0}\right)\right)\right)-\beta\left(V\left(x\left(\tau ; t_{0}, x_{0}, \lambda_{0}\right)\right)\right) \varphi\left(\psi\left(V\left(x\left(\tau ; t_{0}, x_{0}, \lambda_{0}\right)\right)\right)-\varepsilon\right)\right] \\
& \quad+\delta\left(\bar{\alpha}^{-1}\left(V\left(x\left(\tau ; t_{0}, x_{0}, \lambda\right)\right)\right)\right) \geq 0 .
\end{aligned}
$$

This, however, contradicts (24). Hence conditions of the lemma are incompatible with the assumption that the solution $\phi\left(\cdot ; t_{0}, x_{0}, \lambda_{0}\right)$ converges to the origin without leaving the set $\mathcal{D} \times\left[c_{1}, \psi(a)-\varepsilon\right]$ at least once.

When the function $\psi(\cdot)$ is not differentiable it is still possible to provide conditions for specifying solutions escaping a neighborhood of the origin at least once. The conditions are dual to those of Lemma 2, and we present them in the lemma below.

LEMma 4 (solutions escaping the origin 2). Let system (5a) be given and satisfying Assumptions 3 and 4 . Suppose that $\mathcal{D}$ contains the origin. In addition, let the following hold:

(C7) There exist a function $\psi \in \mathcal{K}_{\infty}$ and an $a \in \mathbb{R}_{>0}$ such that for some $\varepsilon \in \mathbb{R}_{>0}$ and all $V \in(0, a]$ the following holds:

$$
\psi(V)[\alpha(V)-\beta(V) \varphi(\psi(V)-\varepsilon)]+V \delta\left(\bar{\alpha}^{-1}(V)\right) \geq 0,
$$

and $\operatorname{hyp}\left(\psi_{[0, a]}\right)$ is star-shaped w.r.t. the origin.

Moreover, let condition (C6) of Lemma 3 hold, and let $\Omega_{a}^{*}$ be the complement of $\Omega_{a}$ in $\overline{\mathcal{D}} \times\left[c_{1}, \psi(a)-\varepsilon\right]$.

Then solutions of $(5 \mathrm{a})$ starting in $\Omega_{a}^{*}$ at $t=t_{0}$ cannot converge to the origin without leaving the set $\overline{\mathcal{D}} \times\left[c_{1}, \psi(a)-\varepsilon\right]$ at least once. 
Proof of Lemma 4. As before, let $\phi\left(\cdot ; t_{0}, x_{0}, \lambda_{0}\right),\left(x_{0}, \lambda_{0}\right) \in \Omega_{a}^{*}$, be a solution of (5a) converging to the origin as $t \rightarrow \infty$ without leaving the set $\overline{\mathcal{D}} \times\left[c_{1}, \psi(a)-\varepsilon\right]$ at least once. Invoking the same argument as in Lemma 3 we can conclude that there is an interval $\left[t^{\prime}, t^{\prime \prime}\right]$ such that $\psi\left(V\left(x\left(t^{\prime} ; t_{0}, x_{0}, \lambda_{0}\right)\right)\right)-\lambda\left(t^{\prime} ; t_{0}, x_{0}, \lambda_{0}\right)-\varepsilon=0$, and for all $t \in\left[t^{\prime}, t^{\prime \prime}\right]$ we have that $\phi\left(t ; t_{0}, x_{0}, \lambda_{0}\right) \in \mathcal{D} \times \Lambda$ and

$$
\psi\left(V\left(x\left(t ; t_{0}, x_{0}, \lambda_{0}\right)\right)\right)-\lambda\left(t ; t_{0}, x_{0}, \lambda_{0}\right)-\varepsilon<0 .
$$

It is clear that if the $\operatorname{set} \operatorname{hyp}\left(\psi_{[0, a]}\right)$ is star-shaped w.r.t. the origin, then the set $\operatorname{hyp}\left(\psi_{[0, a]}-\varepsilon\right)$ is star-shaped w.r.t. the point $(0,-\varepsilon)$.

Consider the function $p:\left[t_{0}, \infty\right) \rightarrow \mathbb{R}:$

$$
p(t)=V\left(x\left(t ; t_{0}, x_{0}, \lambda_{0}\right)\right) /\left(\lambda\left(t ; t_{0}, x_{0}, \lambda_{0}\right)+\varepsilon\right) .
$$

The function $p(\cdot)$ is well defined for $t \in\left[t^{\prime}, t^{\prime \prime}\right]$. Notice that, according to (26), the point $\left(V\left(x\left(t^{\prime} ; t_{0}, x_{0}, \lambda_{0}\right)\right), \lambda\left(t^{\prime} ; t_{0}, x_{0}, \lambda_{0}\right)\right)$ belongs to $\operatorname{hyp}\left(\psi_{[0, a]}-\varepsilon\right)$, and the point $\left(V\left(x\left(t^{\prime \prime} ; t_{0}, x_{0}, \lambda_{0}\right)\right), \lambda\left(t^{\prime \prime} ; t_{0}, x_{0}, \lambda_{0}\right)\right)$ is in the interior of epi $\left(\psi_{[0, a]}-\varepsilon\right)$. Therefore, $p\left(t^{\prime \prime}\right)<p\left(t^{\prime}\right)$, for otherwise the point $\left(V\left(x\left(t^{\prime \prime} ; t_{0}, x_{0}, \lambda_{0}\right)\right), \lambda\left(t^{\prime \prime} ; t_{0}, x_{0}, \lambda_{0}\right)\right)$ would be in $\operatorname{hyp}\left((\psi(V)-\varepsilon)_{[0, a]}\right)$; this would then contradict the condition $\psi\left(V\left(t^{\prime \prime} ; t_{0}, x_{0}, \lambda_{0}\right)\right)-$ $\lambda\left(t^{\prime \prime} ; t_{0}, x_{0}, \lambda_{0}\right)-\varepsilon<0$. On the other hand, there is a $\tau \in\left[t^{\prime}, t^{\prime \prime}\right]$ such that

$$
\begin{aligned}
p\left(t^{\prime \prime}\right)-p\left(t^{\prime}\right)= & \left(t^{\prime \prime}-t^{\prime}\right)\left[\dot{V}\left(x\left(\tau ; t_{0}, x_{0}, \lambda_{0}\right)\right)\left(\lambda\left(\tau ; t_{0}, x_{0}, \lambda_{0}\right)+\varepsilon\right)\right. \\
& \left.-\dot{\lambda}\left(\tau ; t_{0}, x_{0}, \lambda_{0}\right) V\left(x\left(\tau ; t_{0}, x_{0}, \lambda_{0}\right)\right)\right]\left(\lambda\left(\tau ; t_{0}, x_{0}, \lambda_{0}\right)+\varepsilon\right)^{-2} .
\end{aligned}
$$

Hence, invoking Assumption 4, we arrive at the following estimate:

$$
\begin{aligned}
& \dot{V}\left(x\left(\tau ; t_{0}, x_{0}, \lambda_{0}\right)\right)\left(\lambda\left(\tau ; t_{0}, x_{0}, \lambda_{0}\right)+\varepsilon\right)-\dot{\lambda}\left(\tau ; t_{0}, x_{0}, \lambda_{0}\right) V\left(x\left(\tau ; x_{0}, \lambda_{0}\right)\right) \\
& \quad \geq \psi\left(V\left(x\left(\tau ; t_{0}, x_{0}, \lambda_{0}\right)\right)\right) \times\left[\alpha\left(V\left(x ; t_{0}, x_{0}, \lambda_{0}\right)\right)\right)-\beta\left(V\left(x\left(\tau ; t_{0}, x_{0}, \lambda_{0}\right)\right)\right) \\
& \left.\quad \times \varphi\left(\psi\left(V\left(x\left(\tau ; t_{0}, x_{0}, \lambda_{0}\right)\right)\right)-\varepsilon\right)\right]+V\left(x\left(\tau ; t_{0}, x_{0}, \lambda_{0}\right)\right) \delta\left(\bar{\alpha}^{-1}\left(V\left(x\left(\tau ; t_{0}, x_{0}, \lambda_{0}\right)\right)\right) \geq 0 .\right.
\end{aligned}
$$

This implies that $p\left(t^{\prime \prime}\right)-p\left(t^{\prime}\right) \geq 0$, and hence we have reached a contradiction.

4. Examples. In this section we illustrate the theoretical results with examples. We begin with the analysis of systems (1a)-(1c) presented in Example 1 in section 1. Then we proceed to the phase synchronization problem introduced in Example 2. Finally, in section 4.3, we illustrate how Lemma 2 can be used to approach an adaptive control problem for a class of systems nonlinear in the parameters.

4.1. Forward invariant sets and basins of attraction for (1a)-(1c). We start with system (1a):

$$
\begin{aligned}
& \dot{x}=-x+\lambda, \\
& \dot{\lambda}=-\gamma|x|^{3}, \gamma \in \mathbb{R}_{>0} .
\end{aligned}
$$

Let $\mathcal{D}=\mathbb{R}, \Lambda=\mathbb{R}$, and notice that Assumption 1 is satisfied for the first equation in (1a) with $V(x)=x^{2}$, and $\alpha(V)=-2 V, \beta(V)=2 \sqrt{V}, \varphi(|\lambda|)=|\lambda|$. Assumption 2 is fulfilled with $\xi(\cdot) \equiv 0, \delta(|x|)=\gamma|x|^{3}$. Pick a candidate for the function $\psi: \psi(V)=$ $p V, p \in \mathbb{R}_{>0}$, and consider the function $\mathcal{F}: \mathbb{R}_{\geq 0} \rightarrow \mathbb{R}:$

$$
\mathcal{F}(V)=\frac{\partial \psi}{\partial V}(\alpha(V)+\beta(V) \varphi(\psi(V)))+\delta(\sqrt{V})=\left(-2 p+\left(2 p^{2}+\gamma\right) \sqrt{V}\right) V .
$$



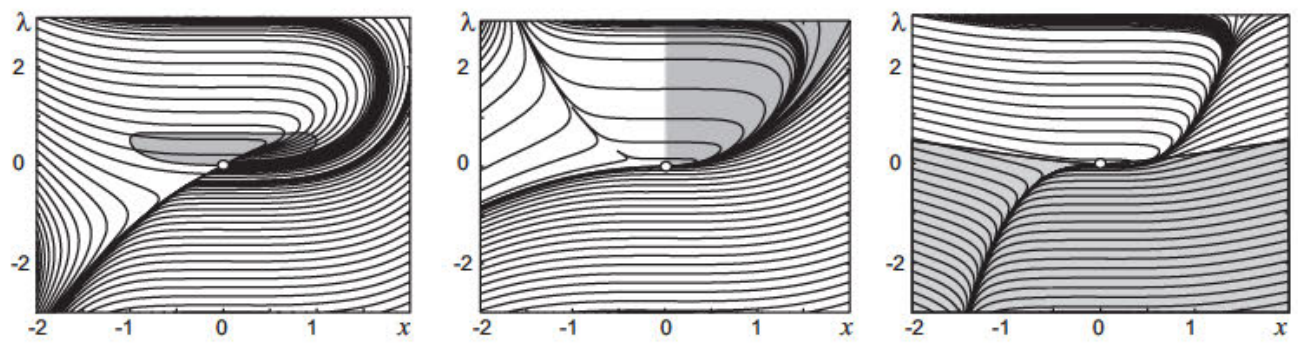

FIG. 4. Left and middle panels: forward invariant sets for systems (1a), (1b) at $\gamma=0.5$ (light grey areas in the plots). The left panel depicts the union of $\Omega_{a}(p), p=(0, \infty)$, defined by $(28)$, superimposed on the phase plot of (1a). The middle panel shows $\Omega_{a}(0.5)$, (29), placed over the phase plot of (1b). The right panel depicts the domain (grey area) corresponding to solutions of (1c) not converging to the origin.

The function is nonpositive for $\sqrt{V} \in\left(0,2 p /\left(2 p^{2}+\gamma\right)\right]$. Finally, note that $\underline{\omega}(\psi(a))=\mathbb{R}$ for all $a>0$. Hence, according to Lemma 1 , the sets

$$
\Omega_{a}(p)=\left\{\left.(x, \lambda)\left|x \in \mathbb{R}, \lambda \in \mathbb{R}, p\left(\frac{2 p}{2 p^{2}+\gamma}\right)^{2} \geq \lambda \geq p\right| x\right|^{2}, p \in \mathbb{R}_{>0}\right\}
$$

are forward invariant. Moreover, $\lim _{t \rightarrow \infty} x\left(t ; x_{0}, \lambda_{0}\right)=0$ for $\left(x_{0}, \lambda_{0}\right) \in \Omega_{a}(p)$. Noticing that the right-hand side of (1a) is locally Lipschitz and using Barbalat's lemma we can conclude that $\lim _{t \rightarrow \infty} \lambda\left(t ; x_{0}, \lambda_{0}\right)=0$. This confirms that the origin is a weak attractor for (1a). An estimate of the forward invariant sets for (1a) is provided in Figure 4. Note also that, since $\mathcal{D}=\mathbb{R}$ and $\Lambda=\mathbb{R}$, Corollary 1 could be applied to this example too.

Let us now proceed with the analysis of system (1b). Its dynamics is described as

$$
\begin{aligned}
& \dot{x}=-x^{2}+\lambda, \\
& \dot{\lambda}=-\gamma|x|^{3}, \gamma \in \mathbb{R}_{>0} .
\end{aligned}
$$

In this particular example we let $\mathcal{D}=\mathbb{R}_{>0}$ and $\Lambda=\mathbb{R}$. One can see that Assumptions 1 and 2 hold for this system with $V=x^{2}, \alpha(V)=-2 V^{3 / 2}, \beta(V)=2 \sqrt{V}, \varphi(|\lambda|)=|\lambda|$, $\xi(\cdot) \equiv 0$, and $\delta(|x|)=\gamma|x|^{3}$. Substituting these into (27) and letting $\psi(V)=p V$ yields $\mathcal{F}(V)=\left(-2 p+2 p^{2}+\gamma\right) V \sqrt{V}$.

It is therefore clear that $\mathcal{F}(V) \leq 0$ as long as $2 p^{2}-2 p+\gamma \leq 0$. Let us suppose that the last inequality holds. Hence condition (C1) of the lemma is satisfied. One can also see that (C2) and (C3) hold too (with $\underline{\omega}(\psi(a))=\mathbb{R}_{\geq 0}$ for all $a>0$ ). Thus Lemma 1 ensures that the sets

$$
\Omega_{a}(p)=\left\{\left.(x, \lambda)|x \in \mathbb{R}, \lambda \in \mathbb{R}, \lambda \geq p| x\right|^{2}, p: 2 p^{2}-2 p+\gamma \leq 0, p \in \mathbb{R}_{>0}\right\}
$$

are forward invariant. Notice that $\gamma=0.5$ satisfies the inequality $2 p^{2}-2 p+\gamma \leq 0$ for $p=0.5$. This is consistent with the phase plots of (1b) in Example 2 (see Figure 1b).

Finally, consider (1c):

$$
\left\{\begin{array}{l}
\dot{x}=-x^{3}+\lambda, \\
\dot{\lambda}=-\gamma|x|^{3}
\end{array}\right.
$$

Let $\mathcal{D}=\mathbb{R}$ and $\Lambda=\mathbb{R}$. The origin of this system is not an attractor. This can be shown by employing the singular transformation $(x, \lambda) \mapsto(\rho, \varphi): x=\rho \cos (\varphi), \lambda=\rho^{2} \sin (\varphi)$ 
followed by the analysis of solutions in a vicinity of the point $(\rho, \varphi)=(0,0)$ in the $(\rho, \varphi)$ coordinates.

Let us see whether we can determine domains corresponding to the solutions of (1c) escaping some specified neighborhoods of the origin. For this purpose we will use Lemma 3. Let $V(x)=|x|^{q}, q \in \mathbb{R}_{>0}$; then Assumption 3 holds with $\alpha(\cdot), \beta(\cdot)$, and $\varphi(\cdot)$ defined as

$$
\alpha(V)=-q V^{\left(1+\frac{2}{q}\right)}, \quad \beta(V)=q V^{\left(1-\frac{1}{q}\right)}, \quad \varphi(|\lambda|)=|\lambda|,
$$

and Assumption 4 holds with $\xi(\cdot) \equiv 0, \delta(|x|)=\gamma|x|^{3}$. Let $\psi(V)=p V^{r}, r \in \mathbb{R}_{>0}$, $p \in \mathbb{R}_{>0}$, and consider

$$
\begin{aligned}
\mathcal{F}(V) & =\frac{\partial \psi}{\partial V}(\alpha(V)-\beta(V) \varphi(\psi(V)-\varepsilon))+\delta\left(V^{\frac{1}{q}}\right) \\
& \geq-p q r V^{\left(r+\frac{2}{q}\right)}-p^{2} q r V^{\left(2 r-\frac{1}{q}\right)}+\gamma V^{\frac{3}{q}} .
\end{aligned}
$$

In order to apply Lemma 3 we need to find $r, q, p \in \mathbb{R}_{>0}$ and $a>0$ such that $\mathcal{F}(V) \geq 0$ for $V \in(0, a]$. Letting $r=1$ and $q=2$ we arrive at $\mathcal{F}(V) \geq-2 p V^{2}-2 p^{2} V^{\frac{3}{2}}+\gamma V^{\frac{3}{2}}$. Hence $\mathcal{F}(V) \geq 0$ whenever $\sqrt{V} \leq(\gamma / 2 p)-p$. Therefore, according to the lemma, solutions of (1c) starting in the complements $\Omega_{a}(p)^{*}$ of

$$
\Omega_{a}(p)=\left\{(x, \lambda) \mid x \in \mathbb{R},\left(\frac{\gamma}{2 p}-p\right)^{2}-\varepsilon \geq \lambda \geq p V-\varepsilon, p \in(0, \sqrt{\gamma / 2})\right\}
$$

in $\mathbb{R} \times\left(-\infty,\left(\frac{\gamma}{2 p}-p\right)^{2}-\varepsilon\right]$ cannot converge to the origin without leaving $\Omega_{a}(p)^{*}$ at least once. The union of $\Omega_{a}(p)^{*}$ over $p$ for $\gamma=0.5$ and $\varepsilon=0.01$ is shown in Figure 4, right panel. Note that in this particular case, since $g(x, \lambda, t)=-\gamma|x|^{3}$ is nonpositive for all $x, \lambda, t \in \mathbb{R}$, solutions starting in this union do not converge to the origin.

4.2. Phase synchronization of neuronal oscillators. We now proceed with the analysis of a somewhat more complicated system: a network of coupled neuronal oscillators (or cells). Interaction between individual elements is allowed to be heterogeneous: the cells are able to interact with immediate neighbors via gap-junctions (intercellular connections enabling direct flows of ions from one to another), or they can transmit pulses to other cells through synaptic connections.

In the absence of coupling, each oscillator generates spikes with a given frequency. The oscillators may not be identical, yet the frequency of oscillations is supposed to be the same for every element in the network. For illustrative purposes we will assume that oscillations in the cells occur through the saddle-node-on-invariant-cycle bifurcation. Such a mechanism is inherent to a wide range of models, including the canonical Hodgkin-Huxley equations describing potential generation in neural membranes via potassium-sodium gates; cf. [14], [19]. Oscillators of this kind have the following normal form [19]:

$$
\dot{x}_{i}=1+x_{i}^{2}+\varepsilon_{i} u(t),
$$

where $x_{i}$ represents the neuron's membrane potential, $\varepsilon_{i} \in \mathbb{R}_{\geq 0}$ is an input gain, and $u: \mathbb{R} \rightarrow \mathbb{R}$ is a function that models the inputs (couplings) applied to the cell. In this model, when $x_{i}\left(\cdot ; t_{0}, x_{0}\right)$ escapes to infinity, a spike is produced and the initial conditions are reset to $-\infty$. The process repeats infinitely many times, giving rise to periodic spikes with infinite amplitude (see Figure 5). 

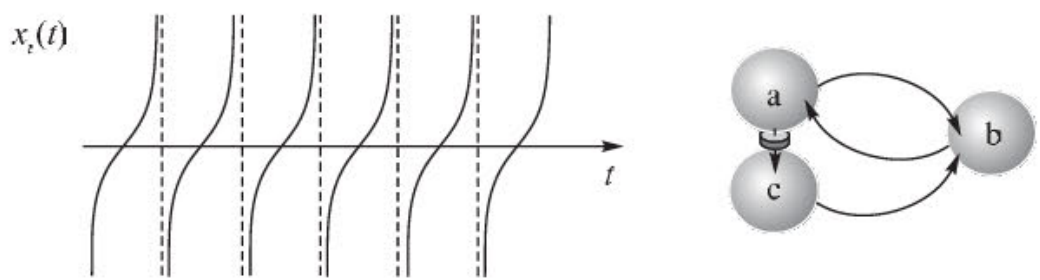

FIG. 5. Networks of phase oscillators. The left panel shows a diagram depicting solutions $x_{i}(t)=-\cot \left(t+\phi_{i}\right), \phi_{i} \in \mathbb{R}, i \in\{a, b, c\}, t \neq \pi k-\phi_{i}, k \in \mathbb{Z}$, produced by the ith equation in the uncoupled system (31)-(33) (i.e., at $\varepsilon=0, \varepsilon_{1}=0$ ) as as function of time. The right panel depicts a diagram of the network described by (31)-(33). Long arrows indicate long-range connections via pulse coupling, and the short arrow indicates the connection between cells a and c via a unidirectional gap junction.

In this particular example we consider a network consisting of three coupled neuronal oscillators, (30), whose topological structure is shown in Figure 5. Neurons $a$ and $b$ interact via bidirectional pulse coupling, neuron $a$ interacts with neuron $c$ via a gap-junction, and $c$ interacts with $a$ synaptically (pulse coupled). Dynamics of such a network can be described as follows [19]:

$$
\begin{aligned}
& \dot{x}_{a}=1+x_{a}^{2}+\varepsilon\left[\delta\left(t-t_{b}\right)+\delta\left(t-t_{c}\right)\right], \\
& \dot{x}_{b}=1+x_{b}^{2}+\varepsilon \delta\left(t-t_{a}\right), \\
& \dot{x}_{c}=1+x_{c}^{2}+\varepsilon_{1}\left(x_{a}-x_{c}\right),
\end{aligned}
$$

where $\delta(\cdot)$ is the Dirac delta function. When $\varepsilon, \varepsilon_{1}$ are sufficiently small, phases of each oscillator can be defined as follows: $\varphi_{i}=t+\phi_{i}, i \in\{a, b, c\}$, where $\phi_{i}$ are "slow" phase fluctuations. Despite the fact that the frequencies of the individual oscillators are kept identical, phase fluctuations, $\phi_{i}$, may vary. The question is whether these fluctuations in the coupled system as functions of $t$ converge to some known values as $t \rightarrow \infty$.

In order to answer this question consider the dynamics of slow phase fluctuations in system (31)-(33) (cf. [19]):

$$
\begin{aligned}
& \dot{\phi}_{a}=\varepsilon / \pi\left[\sin ^{2}\left(\phi_{b}-\phi_{a}\right)+\sin ^{2}\left(\phi_{c}-\phi_{a}\right)\right], \\
& \dot{\phi}_{b}=\varepsilon / \pi \sin ^{2}\left(\phi_{a}-\phi_{b}\right), \\
& \dot{\phi}_{c}=\varepsilon_{1} / 2 \sin \left(2\left(\phi_{a}-\phi_{c}\right)\right) .
\end{aligned}
$$

Denoting $\lambda:=\phi_{b}-\phi_{a}$ and $x:=\phi_{a}-\phi_{c}$ this results in

$$
\begin{aligned}
& \dot{x}=\tilde{\varepsilon}\left[\sin ^{2}(\lambda)+\sin ^{2}(x)\right]-\varepsilon_{1} / 2 \sin (2 x), \tilde{\varepsilon}:=\frac{\varepsilon}{\pi}, \\
& \dot{\lambda}=-\tilde{\varepsilon} \sin ^{2}(x) .
\end{aligned}
$$

Note that (34), (35) locally resemble system (5a). Indeed, the origin of (34) at $\lambda=0$ is locally asymptotically stable in the region $\left\{x \in(-\pi / 2, \pi / 2) \mid \frac{\varepsilon_{1}}{\tilde{\varepsilon}}>\tan (|x|)\right\}$, and the right-hand side of (35) is a nonnegative function of $x, \lambda$.

Consider $V(x)=\tan ^{2}(x)$; then, for all $|x|<M, M=\tan ^{-1}\left|\frac{\varepsilon_{1}}{\tilde{\varepsilon}}\right|, \lambda \in \mathbb{R}$, the following holds: $\dot{V} \leq \alpha(V)+\beta(V) \varphi(|\lambda|)$, where

$$
\begin{aligned}
& \alpha(V)=-2\left(\varepsilon_{1}-\tilde{\varepsilon} V^{1 / 2}\right) V, \\
& \beta(V)=2 \tilde{\varepsilon} V^{1 / 2}(1+V),
\end{aligned} \quad \varphi(|\lambda|)=\left\{\begin{array}{l}
\sin ^{2}(|\lambda|),|\lambda| \in[0, \pi / 2), \\
1,|\lambda| \geq \pi / 2 .
\end{array}\right.
$$


Thus letting $\mathcal{D}=(-\mu, \mu), 0<\mu<M, \Lambda=\mathbb{R}$, and $\underline{\alpha}(|x|)=\bar{\alpha}(|x|)=\tan ^{2}(|x|)$ we can conclude that Assumption 1 holds for (34), (35). With regard to Assumption 2, it is satisfied with $\delta(|x|)=\tilde{\varepsilon} \varphi(|x|), \xi(|\lambda|) \equiv 0$.

Suppose that we wish to apply Lemma 1 . In this case we need to find a strictly monotone function $\psi \in \mathcal{K}$ such that the function $\mathcal{F}(\cdot)$ defined as

$$
\mathcal{F}(V)=\frac{\partial \psi}{\partial V}(\alpha(V)+\beta(V) \varphi(\psi(V)))+\delta\left(\underline{\alpha}^{-1}(V)\right)
$$

is nonpositive in $(0, a]$ for some $a \in \mathbb{R}_{>0}$. Let $\psi(\cdot)$ be such that its restriction on the set $\left\{V \in \mathbb{R}_{\geq 0} \mid V \leq \underline{\alpha}(\mu)\right\}$ is as follows: $\psi(V)=p \tan ^{-1}\left(V^{1 / 2}\right), p \in \mathbb{R}_{>0}$. Then for all $V \in(0, \underline{\alpha}(\mu)]$ the function $\mathcal{F}(\cdot)$ in $(36)$ can be estimated from above as follows:

$$
\begin{aligned}
\mathcal{F}(V) & =p \frac{-2\left(\varepsilon_{1}-\tilde{\varepsilon} V^{\frac{1}{2}}\right) V+2 \tilde{\varepsilon} V^{\frac{1}{2}}(1+V) \sin ^{2}\left(p \tan ^{-1}\left(V^{\frac{1}{2}}\right)\right)}{2 V^{\frac{1}{2}}(1+V)}+\tilde{\varepsilon} \sin ^{2}\left(\tan ^{-1}\left(V^{\frac{1}{2}}\right)\right) \\
& \leq-\frac{p \varepsilon_{1} V^{\frac{1}{2}}}{(1+V)}+\tilde{\varepsilon}\left(p^{3}+p+1\right) V .
\end{aligned}
$$

Hence $\mathcal{F}(V) \leq 0$ whenever $V \leq \tan ^{2}(\mu)$ and $-p \varepsilon_{1}+\tilde{\varepsilon}\left(p^{3}+p+1\right) V^{\frac{1}{2}}(1+V) \leq 0$. Thus solving the latter inequality for $V^{\frac{1}{2}}$ and choosing $V \leq a(p)$, where

$$
a(p)=\min \left\{\tan ^{2}(\mu),\left(r(p)-\frac{1}{3 r(p)}\right)^{2}\right\}, \quad r(p)=\left(\frac{p \varepsilon_{1}+\sqrt{\left(p \varepsilon_{1}\right)^{2}+\frac{4}{2 \tau} \tilde{\varepsilon}^{2}\left(p^{3}+p+1\right)^{2}}}{2 \tilde{\varepsilon}\left(p^{3}+p+1\right)}\right)^{\frac{1}{3}}
$$

we can ensure that condition $(\mathrm{C} 1)$ of the lemma is satisfied. Given that the ball $\left\{x\left|x \in \mathbb{R}^{n},\right| x \mid \leq \tan ^{-1}(a)\right\}$ is contained in $\mathcal{D}$ (condition $(\mathrm{C} 2)$ ) and that $\underline{\omega}(\psi(a))=\mathbb{R}$, Lemma 1 implies that

$$
\Omega_{a}(p)=\left\{(x, \lambda)\left|p \tan ^{-1}\left(a^{\frac{1}{2}}(p)\right) \geq \lambda \geq p\right| x \mid\right\}
$$

is forward invariant. The union of $\Omega_{a}(p), p \in(0,10]$, as well as the phase plot of (34) for $\varepsilon_{1}=0.1, \varepsilon=0.1$ are shown in Figure 6 .

Thus we can conclude that if the values of relative phases $\phi_{a, 0}, \phi_{b, 0}$, and $\phi_{c, 0}$ are chosen so that $\left(\phi_{b, 0}-\phi_{a, 0}, \phi_{a, 0}-\phi_{c, 0}\right) \in \cup_{p>0} \Omega_{a}(p)$, where $\Omega_{a}(p)$ is defined in (37), then the relative phases $\phi_{b}\left(\cdot ; t_{0}, \phi_{a, 0}, \phi_{b, 0}, \phi_{c, 0}\right)-\phi_{a}\left(\cdot ; t_{0}, \phi_{a, 0}, \phi_{b, 0}, \phi_{c, 0}\right)$ and $\phi_{a}\left(\cdot ; t_{0}, \phi_{a, 0}, \phi_{b, 0}, \phi_{c, 0}\right)-\phi_{c}\left(\cdot ; t_{0}, \phi_{a, 0}, \phi_{b, 0}, \phi_{c, 0}\right)$ are bounded on $\left[t_{0}, \infty\right)$. Moreover, $\lim _{t \rightarrow \infty} \phi_{a}(t)-\phi_{c}(t)=0$, and consequently $\lim _{t \rightarrow \infty} \phi_{b}(t)-\phi_{a}(t)=0$. Hence relative phases in the system converge to zero as $t \rightarrow \infty$ provided that $\left(\phi_{b, 0}-\phi_{a, 0}, \phi_{a, 0}-\phi_{c, 0}\right) \in$ $\cup_{p>0} \Omega_{a}(p)$. This corresponds to in-phase synchronization of the solutions of (31)-(33). Looking at the phase plot in Figure 6 one can observe that the actual domain of initial conditions corresponding to in-phase synchronization is substantially larger than that obtained analytically (grey area). This may be viewed as a consequence of choosing functions $V(\cdot), \psi(\cdot), \alpha(\cdot)$, and $\beta(\cdot)$ so that the derivations of $\Omega_{a}(p)$ are kept simple.

4.3. Adaptive control for nonlinearly parameterized systems. As yet another and final illustration consider the following system:

$$
\dot{x}=f(x, \theta, t, u(t)),
$$

where the function $f: \mathbb{R}^{n} \times \mathbb{R}^{m} \times \mathbb{R} \times \mathbb{R}^{q} \rightarrow \mathbb{R}^{n}$ is continuous and locally Lipschitz, $\theta \in \Theta, \Theta \subset \mathbb{R}^{m}$ is the vector of unknown parameters, and $u: \mathbb{R} \rightarrow \mathbb{R}$ is a control 


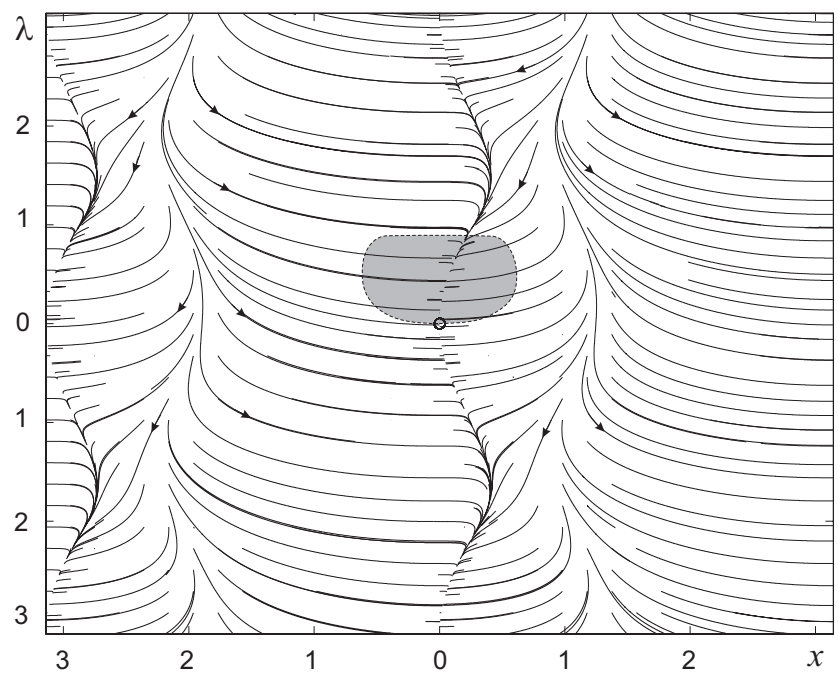

FIG. 6. Estimate of the forward invariant set for system (34), (35) at $\varepsilon_{1}=\varepsilon=0.1$. The estimate (grey shaded area) is the union $\cup_{p \in(0,10]} \Omega_{a}(p)$ of the sets $\Omega_{a}(p)$ defined in (37).

input. With regard to the input $u$, we suppose that it is a continuous function of $x$ and a parameter $\mu \in \mathbb{R}$. Furthermore, we suppose that the for any $\theta \subset \Theta$ there is a stabilizing feedback $u(t)=\tilde{u}(x(t), \mu)$ that is locally Lipschitz in $\mu$. In particular, we suppose that the following assumption holds (cf. [31]).

Assumption 5. Let $\mathcal{M}=\left[\mu_{1}, \mu_{2}\right], \mu_{2}>\mu_{1}$, be an interval in $\mathbb{R}$, and let there exist a positive-definite differentiable function $V: \mathbb{R}^{n} \rightarrow \mathbb{R}$ and two functions $\underline{\alpha}, \bar{\alpha} \in \mathcal{K}_{\infty}$ :

$$
\underline{\alpha}(\|x\|) \leq V(x) \leq \bar{\alpha}(\|x\|) \quad \text { for all } x \in \mathbb{R}^{n} .
$$

The function $f(\cdot, \cdot, \cdot, \cdot)$ in $(38)$ is such that the following hold:

(1) there are known continuous and locally Lipschitz functions $\tilde{u}: \mathbb{R}^{n} \times \mathbb{R} \rightarrow \mathbb{R}^{q}$ and $\alpha \in \mathcal{K}_{\infty}$ such that for any $\theta \in \Theta$ there is a $\mu \in \mathcal{M}$ :

$$
\frac{\partial V}{\partial x} f(x, \theta, t, \tilde{u}(x, \mu)) \leq-\alpha(V(x)) \quad \text { for all } x \in \mathbb{R}^{n}, t \in \mathbb{R}
$$

(2) there is a $\beta \in \mathcal{K}$ such that for every $\mu, \mu^{\prime} \in \mathcal{M}$ and $\theta \in \Theta$ the following holds:

$$
\begin{aligned}
& \frac{\partial V}{\partial x}\left(f(x, \theta, t, \tilde{u}(x, \mu))-f\left(x, \theta, t, \tilde{u}\left(x, \mu^{\prime}\right)\right)\right) \leq \beta(V(x))\left|\mu-\mu^{\prime}\right| \\
& \qquad \text { for all } x \in \mathbb{R}^{n}, t \in \mathbb{R} .
\end{aligned}
$$

Checking that the assumption holds for a given particular system may present certain technical difficulties. Detailed discussion of this issue is outside of the scope of this work.

We are interested in finding a function $\hat{\mu}: \mathbb{R} \rightarrow \mathbb{R}$ such that solutions of $\dot{x}=$ $f(x, \theta, t, \tilde{u}(x, \hat{\mu}(t)))$ converge to the origin as $t \rightarrow \infty$ for all $\theta \in \Theta$. Let

$$
\hat{\mu}=q(h), \quad \dot{h}=-\gamma(\|x\|), \quad \gamma \in \mathcal{K},
$$

where the function $q: \mathbb{R} \rightarrow \mathbb{R}$ is $H$-periodic, that is, $q(h+H)=q(h)$ for all $h \in \mathbb{R}$, and Lipschitz with constant $\ell>0, \max _{h \in \mathbb{R}} q(h)=\mu_{2}, \min _{h \in \mathbb{R}} q(h)=\mu_{1}$. 
Now we are ready to formulate the following result.

Corollary 2. Consider system (38), and let it satisfy Assumption 5. Consider (38), (40):

$$
\dot{x}=f(x, \theta, t, \tilde{u}(x, \hat{\mu})), \quad \hat{\mu}=q(h), \quad \dot{h}=-\gamma(\|x\|),
$$

and suppose that the following conditions hold:

(C8) there is a $V^{*}>0$ such that $\alpha(V)>\beta(V)\left(\mu_{2}-\mu_{1}\right)$ for all $V \geq V^{*}$;

(C9) $\operatorname{epi}\left(\alpha_{\left[0, V^{*}\right]}\right)$ is star-shaped w.r.t. the origin, and the function $\gamma(\cdot)$ in (41) satisfies

$$
\gamma \in \mathcal{K}, \gamma\left(\underline{\alpha}^{-1}(V)\right)<\frac{\alpha(V)^{2}}{4 \beta\left(V^{*}\right) \ell V} \quad \text { for all } V \in \mathbb{R}_{\geq 0} .
$$

Then solutions of (41) are bounded, and furthermore

$$
\lim _{t \rightarrow \infty} x\left(t ; t_{0}, x_{0}, h_{0}\right)=0 \text {, and there is a } \mu^{\prime} \in \mathcal{M}: \lim _{t \rightarrow \infty} \hat{\mu}\left(q\left(t ; t_{0}, x_{0}, h_{0}\right)\right)=\mu^{\prime} .
$$

Proof of Corollary 2. Let $x_{0} \in \mathbb{R}^{n}, h_{0} \in \mathbb{R}$. We begin with showing that solutions of the interconnection are defined for all $t \geq t_{0}$. Given that the right-hand side of (41) is locally Lipschitz, the solution satisfying the initial condition $x\left(t_{0}\right)=x_{0}, h\left(t_{0}\right)=h_{0}$ is defined at least locally in a neighborhood of $\left(x_{0}, h_{0}, t_{0}\right)$. Let $\left[t_{0}, t_{1}\right), t_{1}<\infty$, be the maximal interval of the solution's definition for $t \geq t_{0}$. This implies that for any $M \in$ $\mathbb{R}_{\geq 0}$ there exists a $t^{\prime} \in\left[t_{0}, t_{1}\right)$ such that $\max \left\{\left|h\left(t^{\prime} ; t_{0}, x_{0}, h_{0}\right)\right|,\left\|x\left(t^{\prime} ; t_{0}, x_{0}, h_{0}\right)\right\|\right\} \geq M$. According to Assumption 5, there is a $\mu \in \mathcal{M}$ such that

$$
\begin{aligned}
\dot{V} & \leq \frac{\partial V}{\partial x} f(x, \theta, t, \tilde{u}(x, \mu))+\frac{\partial V}{\partial x}(f(x, \theta, t, \tilde{u}(x, \hat{\mu})-f(x, \theta, t, \tilde{u}(x, \mu)) \\
& \leq-\alpha(V)+\beta(V)|\mu-\hat{\mu}| .
\end{aligned}
$$

Taking condition (C8) of the corollary into account and using the comparison lemma, we conclude that the function $V\left(x\left(\cdot ; t_{0}, x_{0}, h_{0}\right)\right)$ is bounded from above by $\max \left\{V^{*}\right.$, $\left.V\left(x_{0}\right)\right\}$ on $\left[t_{0}, t^{\prime}\right]$. This, however, contradicts $V\left(x\left(t^{\prime} ; t_{0}, x_{0}, h_{0}\right)\right) \geq \underline{\alpha}\left(\| x\left(t^{\prime} ; t_{0}, x_{0}\right.\right.$, $\left.\left.h_{0}\right) \|\right) \geq \underline{\alpha}(M)$ because $M$ can be chosen so that $\underline{\alpha}(M)>\max \left\{V^{*}, V\left(x_{0}\right)\right\}$. Therefore $x\left(\cdot ; t_{0}, x_{0}, h_{0}\right)$ must be bounded on $\left[t_{0}, t_{1}\right)$. On the other hand, according to $(42)$, the following holds:

$$
\left|h\left(t^{\prime} ; t_{0}, x_{0}, h_{0}\right)\right| \leq\left|h_{0}\right|+\left(t^{\prime}-t_{0}\right) \max _{V \in\left[0, \max \left\{V\left(x_{0}\right), V^{*}\right\}\right]}\left\{\frac{\alpha(V)^{2}}{4 \beta\left(V^{*}\right) \ell V}\right\} .
$$

Thus $\left|h\left(\cdot ; t_{0}, x_{0}, h_{0}\right)\right|$ is bounded on $\left[t_{0}, t_{1}\right)$. Hence solutions of (41) are defined for all $t \geq t_{0}$. Furthermore, as follows from (C8), the function $V\left(x\left(\cdot ; t_{0}, x_{0}, h_{0}\right)\right)$ and, consequently, $x\left(\cdot ; t_{0}, x_{0}, h_{0}\right)$ are bounded on $\left[t_{0}, \infty\right)$. Moreover, there is a $t^{*} \geq t_{0}$, independent on $h_{0}$, such that $V\left(x\left(t ; t_{0}, x_{0}, h_{0}\right)\right) \leq V^{*}$ for all $t \geq t^{*}$.

Let us show that $h\left(\cdot ; t_{0}, x_{0}, h_{0}\right)$ is bounded on $\left[t_{0}, \infty\right)$ as well. In order to do so we will invoke Lemma 2. According to Assumption 5 there is a $\mu \in \mathcal{M}$ such that $(\partial V / \partial x) f(x, \theta, t, \tilde{u}(x, \mu)) \leq-\alpha(V)$. Given that the function $q(\cdot)$ in the definition of $\hat{\mu}$ is continuous and periodic, it follows from the intermediate value theorem that there is an $h_{\mu} \in(0, H): q\left(h_{\mu}+n H\right)=\mu$, for all $n \in \mathbb{Z}$. Let us pick an $n$ such that

$$
\eta(n)=h_{\mu}+n H, \quad \eta(n)<h_{0}, \quad h\left(t^{*}, x_{0}, h_{0}\right)-\eta(n) \geq \frac{\alpha\left(V^{*}\right)}{2 \beta\left(V^{*}\right) \ell}
$$


and define $\lambda(t)=h\left(t ; t_{0}, x_{0}, h_{0}\right)-\eta(n)$. It is clear that for $t \geq t^{*}$ the following holds along the solutions of (41):

$$
\dot{V} \leq \frac{\partial V}{\partial x} f(x, \theta, t, \tilde{u}(x, \hat{\mu})) \leq-\alpha(V)+\beta\left(V^{*}\right) \ell|\lambda(t)|, \quad-\gamma\left(\underline{\alpha}^{-1}(V)\right) \leq \dot{\lambda}(t) \leq 0 .
$$

Consider the function $\psi \in \mathcal{K}_{\infty}: \psi(V)=\alpha(V) /\left(2 \beta\left(V^{*}\right) \ell\right)$. According to Lemma 2 and Remark 4 the set

$$
\Omega_{a}=\left\{(x, \lambda) \mid x \in \mathbb{R}^{n}, \lambda \in \mathbb{R}, \psi(a) \geq \lambda \geq \psi(V(x)), V(x) \in[0, a]\right\}
$$

is forward invariant for $t \geq t^{*}$ provided that

$$
\psi(V)\left(-\alpha(V)+\beta\left(V^{*}\right) \ell \psi(V)\right)+V \gamma\left(\underline{\alpha}^{-1}(V)\right) \leq 0 \quad \text { for all } V \in[0, a] .
$$

It is therefore clear that the choice $\gamma\left(\underline{\alpha}^{-1}(V)\right) \leq \frac{\psi(V) \alpha(V)}{2 V}=\frac{\alpha(V)^{2}}{4 \beta\left(V^{*}\right) V \ell}$ ensures that the set $\Omega_{a}$ is forward invariant. Hence every solution of (41) which satisfies the condition $h\left(t^{*} ; t_{0}, x_{0}, h_{0}\right)=\lambda\left(t^{*}\right)+\eta(n), \lambda\left(t^{*}\right)=\frac{\alpha\left(V^{*}\right)}{2 \beta\left(V^{*}\right) \ell}, V\left(x\left(t^{*} ; t_{0}, x_{0}, h_{0}\right)\right) \leq V^{*}$ is bounded on $\left[t^{*}, \infty\right)$. This, in view of the choice of $n$ in the definition of the variable $\lambda(t),(44)$, ensures that $\lambda(\cdot)$ is bounded on $\left[t^{*}, \infty\right)$. Hence $h\left(\cdot ; t_{0}, x_{0}, h_{0}\right)$ is bounded on $\left[t_{0}, \infty\right)$. This implies that $\lim _{t \rightarrow \infty} \int_{t_{0}}^{t} \gamma\left(\left\|x\left(\tau ; t_{0}, x_{0}, h_{0}\right)\right\|\right) d \tau=h^{\prime}<\infty$, where the function $\gamma\left(\left\|x\left(\cdot ; t_{0}, x_{0}, h_{0}\right)\right\|\right)$ is uniformly continuous on $\left[t_{0}, \infty\right)$. Thus, invoking Barbalat's lemma we can conclude that (43) holds.

Note that despite (43) holding, the values of the internal state $h$ of the controller can be large, depending on the initial conditions $x_{0}, h_{0}$. This is a well-known drawback of schemes of this type [15], [31]. One can remove this limitation by replacing (40) with $\hat{\mu}(h)=\mu_{1}+0.5\left(\mu_{2}-\mu_{1}\right)(1+h), \dot{h}=-\gamma(\|x\|) \frac{2 \ell}{\mu_{2}-\mu_{1}} z, \dot{z}=\gamma(\|x\|) \frac{2 \ell}{\mu_{2}-\mu_{1}} h, h\left(t_{0}\right)=$ $h_{0}, z\left(t_{0}\right)=z_{0}, \gamma \in \mathcal{K}$, and restricting the initial condition $h_{0}, z_{0}$ to $h_{0}^{2}+z_{0}^{2}=1$ (see $[39])$. Another issue is that the time needed for $x\left(\cdot ; t_{0}, x_{0}, h_{0}\right)$ to converge to a given neighborhood of the origin may be large too. Derivation of a priori estimates of the convergence times requires further analysis and, possibly, additional constraints.

5. Conclusions. In this manuscript we presented results for finding forward invariant sets and assessing convergence of solutions in dynamical systems with unstable equilibria. In particular, we focused on systems in which stable motions in higherdimensional subspace of the system state space are coupled with unstable motions in a lower-dimensional subspace. Such systems, as has been illustrated with examples, occur in a relevant range of problems, including adaptive control in the presence of general nonlinear parametrization of uncertainty and phase synchronization in networks of coupled oscillators.

Motivated by some limitations of earlier analysis techniques proposed for this class of systems (cf. [39], [15]), we aimed at developing a more versatile alternative. The alternative should apply to systems with unstable attractors while, at the same time, retaining convenience and simplicity of conventional Lyapunov function-based analysis.

The method proposed and discussed in the article allows one to produce simple algebraic tests for finding areas of forward invariance for systems with Lyapunovunstable invariant sets. Moreover, the method can be applied to checking whether a given equilibrium is an attractor, albeit not necessarily stable. Estimates of the attractor basins are also supplied. In addition to convergence and boundedness criteria, geometric intuition behind our results allows one to approach a dual problem: that of 
estimating domains of initial conditions corresponding to solutions escaping a given neighborhood of the origin at least once. These latter results are relevant in the context of determining relaxation times in nonlinear dynamical systems [13].

In spite of the advantages of the method we note that there are limitations too. In particular, we require that the function $V(\cdot)$ characterizing dynamics of the stable part in $(5 \mathrm{a})$ vanish only at a single point, $x=0$. This prevents explicit applications of the results to systems in which solutions of the stable part (in the absence of coupling with the unstable part) are attracted to an orbit or a set which is not a single point. We hope, however, to be able to address this and other issues in future publications.

6. Appendix. Star-shaped sets and envelopes. Let $V$ be a real vector space. In what follows, symbol $[x, y]$ will denote a segment connecting two vectors $x, y \in V:[x, y]=\{\gamma x+(1-\gamma) y \mid \gamma \in[0,1]\}$.

Definition 1. A set $S \subset V$ is star-shaped w.r.t. a point $x \in S$ if for any $y \in S$ the segment $[x, y]$ also belongs to $S:[x, y] \subset S$.

The following properties hold for star-shaped sets in $V$ :

- A set is convex iff it is star-shaped w.r.t. its every point.

- Let $x \in V$ and $W$ be a family of sets star-shaped w.r.t. $x$. Then both the intersection $\cap_{S \in W} S$ and the union $\cup_{S \in W} S$ are star-shaped w.r.t. $x$.

- Let $E$ be a real vector space, $A: V \rightarrow E$ be a linear map, and $S \subset V$ be a star-shaped set w.r.t. a point $x \in S$. Then the image $A(S)$ of $A(\cdot)$ is star-shaped w.r.t. $A(x)$.

DeFinition 2. For any set $D \subset V$ and a point $x \in D$ the star-shaped envelope of $D$ w.r.t. $x, \operatorname{star}_{x}(D)$, is the minimal star-shaped set w.r.t. $x$ which includes D. That is, every star-shaped set w.r.t. $x$ including $D$ must include $\operatorname{star}_{x}(D)$.

Star-shaped envelopes exist and can be defined in two alternative ways: "from above" (as an intersection),

$$
\operatorname{star}_{x}(D)=\bigcap_{S \in W_{x}(D)} S
$$

where $W_{x}(D)$ is a family of sets that are star-shaped w.r.t. $x$ and include $D$; and "from below" (as a union of segments):

$$
S=\bigcup_{y \in D}[x, y] .
$$

Notice that deriving a star-shaped envelope of an analytically defined set computationally is a much easier procedure than that of deriving a convex envelope of the same set.

Let us remind the reader that the epigraph of a real valued function $f: S \rightarrow \mathbb{R}$ is a subset of $S \times \mathbb{R}$ that consists of all points lying on or above its graph: epi $(f)=$ $\{(x, \gamma) \mid x \in S, \gamma \geq f(x)\}$.

Definition 3. The function $f: S \rightarrow \mathbb{R}$ is star-shaped w.r.t. $x \in S$ if $\operatorname{epi}(f)$ is a star-shaped set w.r.t. $(x, f(x))$. The function is convex if it is star-shaped w.r.t. every $x \in S$.

If the function $f: S \rightarrow \mathbb{R}$ is star-shaped w.r.t. $x \in S$, then $S$ must necessarily be star-shaped w.r.t. $x$. Alternatively, we can use the following definition.

Definition 4. A function $f: S \rightarrow \mathbb{R}$ (on a star-shaped set $S$ w.r.t. $x$ ) is starshaped w.r.t. $x$ if the following holds for any $y \in S$ and every $\gamma \in[0,1]$ :

$$
f(\gamma x+(1-\gamma) y) \leq \gamma f(x)+(1-\gamma) f(y)
$$


Expression (47) is a form of Jensen's inequality with one "fixed end." It is obvious that a function on $S$ is convex iff it is star-shaped w.r.t. every point $x \in S$.

DEFINITION 5. Let $f: S \rightarrow \mathbb{R}$ be a bounded from above and from below function $(A<f(x)<B)$. The supremum of star-shaped (w.r.t. $x)$ minorants of $f(\cdot)$ is the star-shaped envelope of $f(\cdot)$ w.r.t. $x$, $\operatorname{star}_{x}(f)$.

Note that Definition 5 can be viewed as a definition of the star-shaped envelope of a function "from above" (compare with (45)).

Let $S \subset V$ be a star-shaped set w.r.t. $x \in S$, and let $\operatorname{conv}(f)$ be the convex envelope of $f(\cdot)$. The following properties hold for the star-shaped sets and functions: $\operatorname{conv}(f)(y) \leq \operatorname{star}_{x}(f)(y) \leq f(y)$. The first property follows immediately from the definition, and the second property is a consequence of the following fact:

$$
\operatorname{epi}(f) \subset \operatorname{epi}\left(\operatorname{star}_{x}(f)\right) \subset \operatorname{epi}(\operatorname{conv}(f)) .
$$

Let us follow the definition of a star envelope of a set from below, (46), and produce the definition of a star envelope of a function "from below." Consider the one-dimensional case: $V=\mathbb{R}$. Let $S=[a, b], x \in S$; for any $z \in S(z \neq x)$ we define

$$
\phi_{z, x}(y)=\left\{\begin{array}{l}
\min \left\{f(y), \frac{y-x}{z-x} f(x)+\frac{z-y}{z-x} f(z)\right\} \text { if } 0<\frac{y-x}{z-x}<1 \\
f(y) \text { else. }
\end{array}\right.
$$

As follows from Jensen's inequality with one fixed end, (47), the following holds:

$$
\operatorname{star}_{x}(f)(y)=\inf _{z \in S}\left\{\phi_{z, x}(y)\right\}
$$

One can also see that the definitions of star-shaped envelopes of a function "from above" and "from below" are equivalent.

The following proposition is obvious.

Proposition 1. Let $S=[a, b]$ be a closed interval in $\mathbb{R}, p$ be an element from $S$, and $f: S \rightarrow \mathbb{R}$ be a continuous function. Consider the functions $\phi_{z, p}(\cdot), z \in S$, defined as in (49). Then the following hold:

(1) the functions $\phi_{z, p}(\cdot), p \in S$, are equicontinuous with $f(\cdot)$;

(2) the set of functions $\left\{\phi_{z, p}(\cdot)\right\}, p \in S$, is compact;

(3) the function $\operatorname{star}_{p}(f), p \in S$, exists and is a continuous function;

(4) if a continuous function on $S$ achieves its minimum at a single point $x$, then its star-shaped envelope w.r.t. $x$ has the same property;

(5) the star-shaped envelope (w.r.t. a point $p \in S$ ) of a monotone function is monotone.

The first property follows immediately from (49). Properties 2 and 3 follow from the Arzela-Ascoli theorem. Indeed, according to this, equicontinuity and uniform boundedness of $\phi_{z, p}(\cdot), p \in S$, imply that $\left\{\phi_{z, p}(\cdot)\right\}$ is relatively compact. Compactness then follows from the fact that the set of functions $\left\{\phi_{z, p}(\cdot)\right\}$ is closed. To demonstrate the existence and continuity of $\operatorname{star}_{p}(f), p \in S$, consider a sequence $\left\{g_{i}\right\}_{i=1}^{\infty}$ of grids $g_{i}=\{a, a+(b-a) / i, a+(b-a) k / i, \ldots, b\}, 1 \leq k<i$ on $S$, and define

$$
f_{i}(y)=\inf _{r \in g_{2 i}}\left\{\phi_{r, p}(y)\right\} .
$$

The sequence of functions $\left\{f_{i}(\cdot)\right\}_{i=1}^{\infty}$ is equicontinuous, and $f_{1}(\cdot) \geq f_{2}(\cdot) \geq \cdots \geq$ $f_{n}(\cdot) \geq \cdots$ pointwise. This means that the sequence converges uniformly and that 
$\lim _{i \rightarrow \infty} f_{i}(y)=\inf _{z \in S}\left\{\phi_{z, p}(y)\right\}=\operatorname{star}_{p}(f)(y)$. Continuity of the limiting function, $\operatorname{star}_{p}(f)$, follows from equicontinuity of the family $\left\{f_{i}(\cdot)\right\}_{i=1}^{\infty}$. Property 4 is easily verifiable by the contradiction argument. Property 5 is the consequence of the fact that the functions $f_{i}(\cdot)$ are monotone (by construction) if the function $f(\cdot)$ is monotone.

Let $V=R^{n}$, and let $S \subset V$ be compact and star-shaped w.r.t. $x$. For every $z \in S$ and $\gamma \in[0,1]$ we define $\psi_{z}(\gamma)=\min \{f((1-\gamma) x+\gamma z),(1-\gamma) f(x)+\gamma f(z)\}$. Then

$$
\operatorname{star}_{x}(f)(y)=\inf _{(1-\gamma) x+\gamma z=y, \gamma \in[0,1], z \in S}\left\{\psi_{z}(y)\right\} .
$$

The properties of $\operatorname{star}_{x}(f)(y)$ depend on the properties of mapping

$$
y \mapsto\{(\gamma, z) \mid(1-\gamma) x+\gamma z=y, \gamma \in[0,1], z \in V\} .
$$

If this mapping is continuous (in the Hausdorff metrics in the space of compact sets), then the star-shaped envelope of every continuous in $S$ function w.r.t. $x$ is also continuous. Mapping (50) is continuous in $S$ iff the Minkovski functional

$$
p(y)=\inf _{a>0}\{a \mid y-x \in a(S-x)\}
$$

is continuous in $S$.

\section{REFERENCES}

[1] F. Allgower, J. Ashman, and A. Ilchmann, High-gain adaptive $\lambda$-tracking for nonlinear systems, Automatica, 33 (1997), pp. 881-888.

[2] A. Andronov, E. Leontovich, I. Gordon, And A.G. MaǏer, Qualitative Theory of SecondOrder Dynamic Systems, Halsted Press, New York, 1973.

[3] J.-P. Aubin, Viability Theory, Birkhäuser, Boston, 1991.

[4] J.-P. Aubin, Viability solutions to structured Hamilton-Jacobi equations under constraints, SIAM J. Control Optim., 49 (2011), pp. 1881-1915.

[5] E. BARBASHin AND Y. Alimov, On the theory of dynamical systems with multi-valued and discontinuous characteristics, Dokl. Akad. Nauk SSSR, 140 (1961), pp. 9-11.

[6] N. Bautin And E. Leontovich, Methods and Means for a Qualitative Investigation of Dynamical Systems on the Plane, Nauka, Moscow, 1990.

[7] A. Bermant, A Course of Mathematical Analysis: Part I, Pergamon Press, New York, 1963.

[8] O. BogoyavlenskiI, Metody Kachestvennoi Teorii Dinamicheskikh System v Astrophisike I Gazovoi Dinamike, Nauka, Moscow, 1980.

[9] N. Chetaev, The Stability of Motion, Pergamon Press, New York, 1961.

[10] J. EnGland, Stability in topological dynamics, Pacific J. Math., 20 (1967), pp. 479-485.

[11] D. Fairhurst, I. Tyukin, H. Nijmeijer, And C. van Leeuwen, Observers for canonic models of neural oscillators, Math. Model. Nat. Phenom., 5 (2010), pp. 146-194.

[12] M. Goman And M. Demenkov, Multiple attractor dynamics in active flutter suppression problem, in Proceedings of the ICNPAA 2008: Mathematical Problems in Engineering, Aerospaces and Sciences, Genoa, Italy, 2008.

[13] A. Gorban, Singularities of Transition Processes in Dynamical Systems: Qualitative Theory of Critical Delays, Electron. J. Differ. Equ. Monogr. 5, Texas State University-San Marcos, San Marcos, TX, 2004; also available online from http://ejde.math.txstate.edu/ Monographs/05/.

[14] A. Hodgkin And A. Huxley, A quantitative description of membrane current and its application to conduction and excitation in nerve, J. Physiol., 117 (1952), pp. 500-544.

[15] A. Ilchmann, Universal adaptive stabilization of nonlinear systems, Dynam. Control, 7 (1997), pp. 199-213.

[16] A. Ilchmann and E. RYan, On gain adaptation in adaptive control, IEEE Trans. Automat. Control, 48 (2003), pp. 895-899.

[17] A. Ilchmann and S. Townley, Adaptive sampling control of high-gain stabilizable systems, IEEE Trans. Automat. Control, 44 (1999), pp. 1961-1966. 
[18] A. Isidori, Nonlinear Control Systems: An Introduction, 2nd ed., Springer-Verlag, Berlin, 1989.

[19] E. M. Izhikevich, Dynamical Systems in Neuroscience, MIT Press, Cambridge, MA, 2007.

[20] P. Kloeden and M. Rasmussen, Nonautonomous Dynamical Systems, Math. Surveys Monogr. 176, American Mathematical Society, Providence, RI, 2011.

[21] A. Kolmogorov, Sulla teoria di Volterra della lotta per l'esistenza, Giornale Instituto Ital. Attuari, 7 (1936), pp. 74-80.

[22] L. LuUng, System Identification: Theory for the User, Prentice-Hall, Englewood Cliffs, NJ, 1999.

[23] A. Lyapunov, The General Problem of the Stability of Motion, Kharkov Mathematical Society, Kharkiv, Ukraine, 1892 (in Russian).

[24] I. MASUBUCHI, Analysis of positive invariance and almost regional attraction via density functions with converse results, IEEE Trans. Automat. Control., 52 (2007), pp. 1329-1333.

[25] V. Matrosov, On the stability of motion, J. Appl. Math. Mech., 26 (1963), pp. 1337-1353.

[26] J. Milnor, On the concept of attractor, Comm. Math. Phys., 99 (1985), pp. 177-195.

[27] A. Monse, Control using logic-based switching, in Trends in Control, Springer-Verlag, Berlin, 1995, pp. 69-113.

[28] M. NaGumo, Über die lage der integralkurven gewöhnlicher differentialgleichungen, Proc. Phys. Math. Soc. Japan, 24 (1942), pp. 551-559.

[29] K. S. Narendra And A. M. Annaswamy, Stable Adaptive Systems, Prentice-Hall, Englewood Cliffs, NJ, 1989.

[30] H. Nijmeijer And A. van der Schaft, Nonlinear Dynamical Control Systems, Springer-Verlag, New York, 1990.

[31] J.-B. Pomet, Remarks on sufficient information for adaptive nonlinear regulation, in Proceedings of the 31st IEEE Conference on Decision and Control, 1992, pp. 1737-1739.

[32] M. I. Rabinovic, R. Huerta, P. Varona, and V. Aframovich, Transient cognitive dynamics, metastability, and decision-making, PLoS Comput. Biol., 4 (2008), pp. 1-9.

[33] M. I. Rabinovic, P. Varona, A. Selverston, and H. Abarbanel, Dynamical principles in neuroscience, Rev. Modern Phys., 78 (2006), pp. 1213-1265.

[34] A. Rantzer, A dual to Lyapunov's stability theorem, Systems Control Lett., 42 (2001), pp. 161168.

[35] M. Timme, F. Wolf, And T. Geisel, Prevalence of unstable attractors in networks of pulsecoupled oscillators, Phys. Rev. Lett., 89 (2002), 154105.

[36] M. Timme, F. Wolf, And T. Geisel, Unstable attractors induce perpetual synchronization and desynchronization, Chaos, 3 (2003), pp. 377-387.

[37] S. TownlEy, Topological aspects of universal adaptive stabilization, SIAM J. Control Optim., 34 (1996), pp. 1044-1070.

[38] I. TYUKIn AND D. FAIRHURST, Making sense of unstable convergence in the problem of adaptive observer design, in Proceedings of the 18th IFAC World Congress on Automatic Control, 2011.

[39] I. Tyukin, E. Steur, H. Nijmeijer, And C. van Leeuwen, Nonuniform small-gain theorems for systems with unstable invariant sets, SIAM J. Control Optim., 47 (2008), pp. 849-882.

[40] I. VADIA AND M. P.G., Lyapunov measure for almost everywhere stability, IEEE Trans. Automat. Control., 53 (2008), pp. 307-323.

[41] V. Zubov, Methods of A.M. Lyapunov and Their Applications, P. Noordhoff, Ltd., Groningen, The Netherlands, 1964. 